\title{
Estimation of High Structural Reliability Involving Nonlinear Dependencies Based on Linear Correlations
}

\author{
Liulin Kong, ${ }^{1}$ Heng Li $\mathbb{D}^{2},{ }^{2}$ Bo Zhang, ${ }^{2,3}$ and Hanbin Luo ${ }^{4}$ \\ ${ }^{1}$ Faculty of Engineering, China University of Geosciences (Wuhan), Wuhan, Hubei 430074, China \\ ${ }^{2}$ Department of Building and Real Estate, The Hong Kong Polytechnic University, Kowloon, Hong Kong \\ ${ }^{3}$ School of Mechanics and Engineering, Applied Mechanics and Structure Safety Key Laboratory of Sichuan Province, \\ Southwest Jiaotong University, Chengdu, China \\ ${ }^{4}$ Department of Construction Management, School of Civil Engineering and Mechanics, \\ Huazhong University of Science and Technology, Wuhan, Hubei 430074, China
}

Correspondence should be addressed to Heng Li; bshengli@polyu.edu.hk

Received 11 September 2020; Revised 12 July 2021; Accepted 19 July 2021; Published 25 August 2021

Academic Editor: Gang Zhou

Copyright (c) 2021 Liulin Kong et al. This is an open access article distributed under the Creative Commons Attribution License, which permits unrestricted use, distribution, and reproduction in any medium, provided the original work is properly cited.

Stochastic nonlinear dependencies have been reported extensively between different uncertain parameters or in their time or spatial variance. However, the description of dependency is commonly not provided except a linear correlation. The structural reliability incorporating nonlinear dependencies thus needs to be addressed based on the linear correlations. This paper first demonstrates the capture of nonlinear dependency by fitting various bivariate non-Gaussian copulas to limited data samples of structural material properties. The vine copula model is used to enable a flexible modeling of multiple nonlinear dependencies by mapping the linear correlations into the non-Gaussian copula parameters. A sequential search strategy is applied to achieve the estimate of numerous copula parameters, and a simplified algorithm is further designed for reliability involving stationary stochastic processes. The subset simulation is then adopted to efficiently generate random variables from the corresponding distribution for high reliability evaluation. Two examples including a frame structure with different stochastic material properties and a cantilever beam with spatially variable stochastic modulus are investigated to discuss the possible effects of nonlinear dependency on structural reliability. Since the dependency can be determined qualitatively from limited data, the proposed method provides a feasible way for reliability evaluation with prescriptions on correlated stochastic parameters.

\section{Introduction}

Reliability analysis of structural systems involves the distribution modeling of a random vector $\mathrm{X}=\left[X_{1}, X_{2}, \ldots, X_{n}\right]$ or a stochastic process $\mathrm{X}(t)=\left[X\left(t_{1}\right), X\left(t_{2}\right), \ldots, X\left(t_{n}\right)\right]$, which is used to represent the inherent uncertainties in material properties, geometrical dimensions, loading conditions, or their time/spatial variance in engineering practice [1]. The failure probability of a structural system is defined as the integral of the joint probability density function (PDF) $f_{\mathrm{X}}(\mathrm{X})$ of the random vector $\mathrm{X}$ over the failure domain defined by the performance function $G(\mathrm{X}) \leq 0$ :

$$
p_{f}=\int_{G(\mathrm{X}) \leq 0} f_{\mathrm{X}}(\mathrm{X}) \mathrm{dX} \text {. }
$$

Equation (1) implies the need of a joint probability distribution for estimating failure probability. If the random variables are mutually independent, the joint probability distribution will be a simple multiplication of its marginal distributions. However, correlations may exist between different stochastic parameters or in the variance of a stochastic parameter over time or space. For example, correlations are reported between wind speed and wind direction or between Young's modulus and Poisson's ratio [2, 3]. The time-dependent or spatially variable stochastic parameter (e.g., time series of external loads, material modulus varying along space, or variation of pore structure in radial distribution $[4,5])$ that is often characterized by a stochastic process can also be viewed as correlated random variables 
with high dimensions [6-9]. Ignoring these correlations will lead to a biased estimate of the structure reliability.

In structural reliability engineering practice, the probabilistic description of uncertain parameters in terms of univariate distributions and pairwise correlations is ubiquitous. The dependency that shapes the scatter of data points is commonly assumed linear for convenience (e.g., [10, 11]). However, the validity of this assumption is questioned and the corresponding model approximation is coarse if the dependency is nonlinear [3]. In other words, reliability evaluation involving nonlinear data dependencies by linear transformations (e.g., the orthogonal and Nataf transformation) can be inaccurate [12].

The copula theory provides a feasible way of characterizing nonlinear dependencies [13]. It has been widely applied to reliability problems where the data scatter exhibits nonlinear characteristics. For instance, the torsional moment and external forces can be upper tail dependent that is better captured by a Gumbel copula [14]. The time-variant peaks of snow load can also show the Gumbel copula characteristics [15]. For a given copula, the distribution model is usually established by matching it to the correlations of uncertain parameters $[16,17]$. There are three major measures of correlations, namely, Pearson's rho, Spearman's rho, and Kendall's tau [18]. The first measure is known as the linear correlation while the other two are the rank correlations. Retrieving copula parameters from the rank correlations is relatively straightforward because the estimate is independent of the marginal distributions [18]. However, the use of linear correlation coefficient is a more common practice in the literature [15]. Compared to the rank correlations, the retrieval of copula parameters from the linear correlations is more complicated because the estimate depends on both the copula function and the marginal distributions [18]. Although some efficient quadrature techniques can be adopted to solve the mathematics $[16,17]$, the proposed approach to the retrieval of non-Gaussian copula parameters from linear correlation coefficients are restricted to the correlated bivariate cases $[16,17,19]$. The difficulty of generalization to the correlated multivariate cases is rooted in the inflexibility of multivariate nonelliptical copulas for representing multiple nonlinear dependencies [20].

The multiple nonlinear dependencies thus need to be addressed, particularly when the linear correlations of stochastic parameters are ubiquitous in the literature. In this paper, a vine copula approach is adopted for multiple dependence modeling. The copula parameters are retrieved from the univariate distributions and correlation matrix via a sequential search strategy (S3) [21, 22]. In [21], a simplified version of S3 was particularly designed for parameter retrieval in reliability involving stationary stochastic processes (i.e., spatial-variant reliability). Once the joint probability distribution is represented in terms of vine copulas, the isoprobabilistic transformation for nonlinear dependent random variable can be integrated into the reliability approach. Since the expected reliability for structures are often high, the subset simulation (SS) is adopted to estimate the failure probability. The main objectives are to demonstrate the possible nonlinear dependencies in practice and assess its impact on high structural reliability. To this end, Section 2 briefly reviews the copula theory and shows the possibly nonlinear dependencies underlying the stochastic structural material properties. The vine copula approach with the copula parameters retrieved from univariate distributions and correlation matrix by S3 is provided in Section 3. The SS is then extended to nonlinear dependences where the correlated Gaussian/non-Gaussian is targeted, as shown in Section 4. Section 5 uses two examples of finite element (FE) analysis - a frame structure with different stochastic material properties and a cantilever beam with spatially variable stochastic modulus-to discuss the possible effects of nonlinear dependence on structural reliability. Finally, conclusions are presented in Section 6.

\section{Nonlinear Dependencies in Structural Material Properties}

A copula is an $n$-dimensional cumulative distribution functions $(\mathrm{CDF}) \mathrm{C}:[0,1]^{n} \longrightarrow[0,1]$ such that each of its marginal CDFs is uniform on the interval $[0,1]$ [13]. Copulas can model the dependence between random variables with arbitrary marginal distributions and link multivariate distributions functions of random variables to their univariate marginal distributions. Let $F_{i}\left(x_{i}\right), i=1, \ldots, n$, denote the marginal CDFs of $\mathbf{X}$, and the joint probability distribution of $\mathbf{X}$ can be expressed as

$$
\begin{aligned}
F_{\mathrm{X}}(\mathrm{X}) & =C\left(F_{1}\left(x_{1}\right), \ldots, F_{n}\left(x_{n}\right) ; \theta\right) \\
& =C\left(u_{1}, \ldots, u_{n}\right),
\end{aligned}
$$

where $\mathrm{u}=\left(u_{1}, \ldots, u_{n}\right)$ denotes a dependent, uniformly distributed vector of random variables and $u_{i}=F_{i}\left(x_{i}\right), \theta$ denotes the set of parameters for the copula, and $C(\mathrm{u})$ is the copula function, which can be derived as

$$
C(\mathrm{u})=F\left(F_{1}^{-1}\left(u_{1}\right), \ldots, F_{n}^{-1}\left(u_{n}\right)\right)
$$

where $F_{i}^{-1}\left(u_{i}\right)$ is the inverse function of the corresponding marginal distributions.

In structural systems, the linear dependence characterized by a Gaussian copula is usually assumed for correlated random variables (e.g., the Nataf transformation). In this section, the validity of the Gaussian assumption is examined through experimental data from standard tensile tests of low carbon steels. The low carbon steels are widely used materials in structures and they have various types. Here, a batch of low carbon steels (grade Q235 in Chinese Standard GB/T 17107-1997, equivalently ISO Fe360 [23]) collected from the same construction site is used for experiments. It is known that there exists dependencies between diameter $D$ and yield load $Y L$ and between yield load $Y L$ and extreme load EL. The best-fit copula to the measured dependence structure can be identified based on the minimum value of the Akaike information criterion (AIC) and the Bayesian information criterion (BIC) [16], which are calculated, respectively, as 


$$
\begin{aligned}
& \mathrm{AIC}=-2 \sum_{i=1}^{N} \ln c\left(u_{1 i}, u_{2 i}\right)+2 k, \\
& \mathrm{BIC}=-2 \sum_{i=1}^{N} \ln c\left(u_{1 i}, u_{2 i}\right)+k \ln N
\end{aligned}
$$

where $N$ is the sample size, $k$ is the number of copula parameters, $c_{1,2}\left(u_{1}, u_{2}\right)=\partial C\left(u_{1}, u_{2}\right) / \partial u_{1} \partial u_{2}$ is the density function of a bivariate copula, and $\left(u_{1 i}, u_{2 i}\right),\left(u_{2 i}, u_{3 i}\right)$, and $\left(u_{1 i}, u_{3 i}\right)$ are the coordinates in the unit square space (Figures 1(b), 1(d), and 1(f), respectively) transformed from the measured data in the original space (Figures 1(a), 1(c), and $1(\mathrm{e}))$, which is defined as

$$
\left\{\begin{array}{l}
u_{1 i}=\frac{\operatorname{rank}\left(D_{i}\right)}{N+1}, \\
u_{2 i}=\frac{\operatorname{rank}\left(Y L_{i}\right)}{N+1}, i=1,2, \ldots, N \\
u_{3 i}=\frac{\operatorname{rank}\left(E L_{i}\right)}{N+1}
\end{array}\right.
$$

where rank (.) denotes the rank of $D_{i}, Y L_{i}$ and $E L_{i}$ among the set $\left\{D_{1}, \ldots, D_{N}\right\},\left\{Y L_{1}, \ldots, Y L_{N}\right\}$, and $\left\{E L_{1}, \ldots, E L_{N}\right\}$, respectively. For illustrative purposes, the Gaussian copula, Clayton copula, CClayton copula, and Frank copula, as listed in Table 1, are examined in this paper. The AIC and BIC values for the four copulas are presented in Table 2 .

Although the materials are collected from the same batch, the uncertainty of material properties as a result of manufacturing error is observed. Results from Table 2 indicate that the Clayton copula is the best-fit copula for modeling the dependence between $D$ and $Y L$ and between $D$ and $E L$, while the Frank copula is the best-fit copula for modeling the dependence between $Y L$ and EL. In other words, none of the dependencies are linear. Thus, the incorporation of nonlinear dependences into structural reliability is necessary. However, the above copulas are fitted only for the bidimensional marginal distributions, while the reliability evaluation requires the joint probability distribution (i.e., the trivariate distribution of $D, Y L$, and $E L$ ). The construction of the multivariate distribution that involves the above bidimensional marginal distributions requires a hierarchical nesting of the bivariate copulas, which will be presented in Section 3.

\section{Pair-Copula Decomposition Approach to Multivariate Distribution Construction from Marginals and Linear Correlations}

3.1. Multivariate Probability Distribution Construction by Pair-Copula Decomposition. A multivariate copula is needed for modeling the multivariate probability distribution. However, the construction of an $n$-dimensional copula while maintaining high flexibility is challenging due to the compatibility problem [13]. A promising way to solve this problem is the pair-copula decomposition approach [24].

According to Aas et al. [24], the $n$-dimensional joint PDF of $\mathbf{X}$ can be decomposed as

$$
f_{\mathrm{X}}(\mathrm{X})=f\left(x_{1}\right) f\left(x_{2} \mid x_{1}\right) \ldots f\left(x_{n} \mid x_{1}, \ldots, x_{n-1}\right),
$$

where the conditional PDF in the form of $f(x \mid \mathrm{v})$ can be represented as

$$
f(x \mid \mathrm{v})=c_{x, v_{j} \mid \mathrm{v}_{-j}}\left(F\left(x \mid \mathrm{v}_{-j}\right), F\left(v_{j} \mid \mathrm{v}_{-j}\right)\right) f\left(x \mid \mathrm{v}_{-j}\right),
$$

where $v_{j}$ is an arbitrarily selected element of $\mathrm{v}$ while $v_{-j}$ denotes the vector $\mathrm{v}$ excluding $v_{j}$ and $c(\cdot)$ is the density function of $C(\cdot)$. Using equation (7) recursively, a multivariate joint PDF can be represented by a product of paircopula density functions, acting on several different conditional probability distributions. The pair-copula construction involves marginal conditional distributions in the form of $F(x \mid \mathrm{v})$, which can also be recursively expressed in terms of the partial derivative of a bivariate copula with respect to the second parameter [25]:

$$
\begin{aligned}
F(x \mid \mathrm{v}) & =\frac{\partial C\left(F\left(x \mid \mathrm{v}_{-j}\right), F\left(v_{j} \mid \mathrm{v}_{-j}\right) ; \theta\right)}{\partial F\left(v_{j} \mid \mathrm{v}_{-j}\right)} \\
& =h_{x \mid v_{j}: \mathrm{v}_{-j}}\left(F\left(x \mid \mathrm{v}_{-j}\right), F\left(v_{j} \mid \mathrm{v}_{-j}\right)\right) .
\end{aligned}
$$

If $\mathrm{v}$ is a univariate, equation (8) can be simplified as follows:

$$
\begin{aligned}
F\left(x_{2} \mid x_{1}\right) & =\frac{\partial C\left(F_{2}\left(x_{2}\right), F_{1}\left(x_{1}\right)\right)}{\partial F_{1}\left(x_{1}\right)} \\
& =h_{2 \mid 1}\left(u_{2}, u_{1}\right) .
\end{aligned}
$$

For high-dimensional distributions, there are generally many different representations of $f_{\mathrm{X}}(\mathrm{X})$ using the paircopula constructions. The vines are commonly used to organize the decompositions [26]. The C-vine and D-vine [27] are two special cases of regular vines, as shown in Figure 2.

For an $n$-dimensional problem, both $\mathrm{C}$-vine and $\mathrm{D}$-vine consist of $n-1$ trees $T_{j}, j=1, \ldots, n-1$. Tree $T_{j}$ has $n+1-j$ nodes and $n-j$ edges. Each edge corresponds to a paircopula density function (e.g., edge $1, n-1 \mid 2, \ldots, n-2$ corresponds to the copula function $\left.c_{1, n-1 \mid 2, \ldots, n-2}(\cdot)\right)$ and the edge labels the nodes to be drawn. The edges in tree $T_{j+1}$ are determined only by the nodes in tree $T_{j}$. The way of connecting nodes in $\mathrm{C}$-vines and $\mathrm{D}$-vines is different. In a $\mathrm{D}$-vine, each node is connected by no more than two edges. In a $\mathrm{C}$-vine, each tree $T_{j}$ has a unique node that is connected by the other $n-j$ edges.

$f_{\mathrm{X}}(\mathrm{X})$ corresponding to a D-vine and C-vine is, respectively, given as 


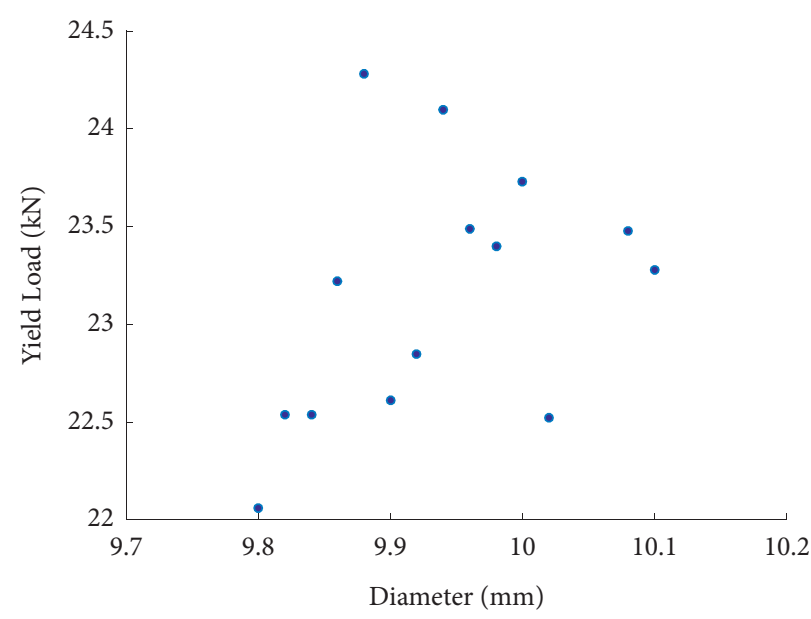

(a)

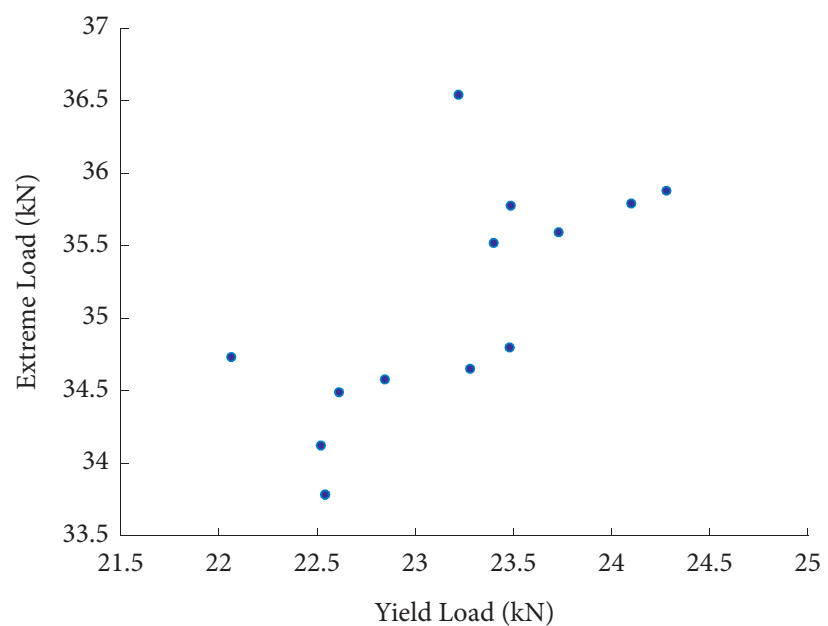

(c)

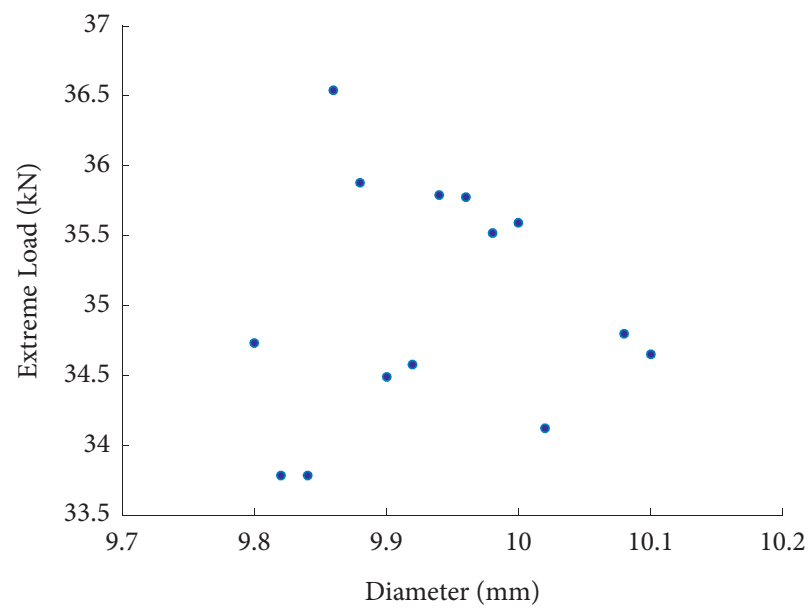

(e)

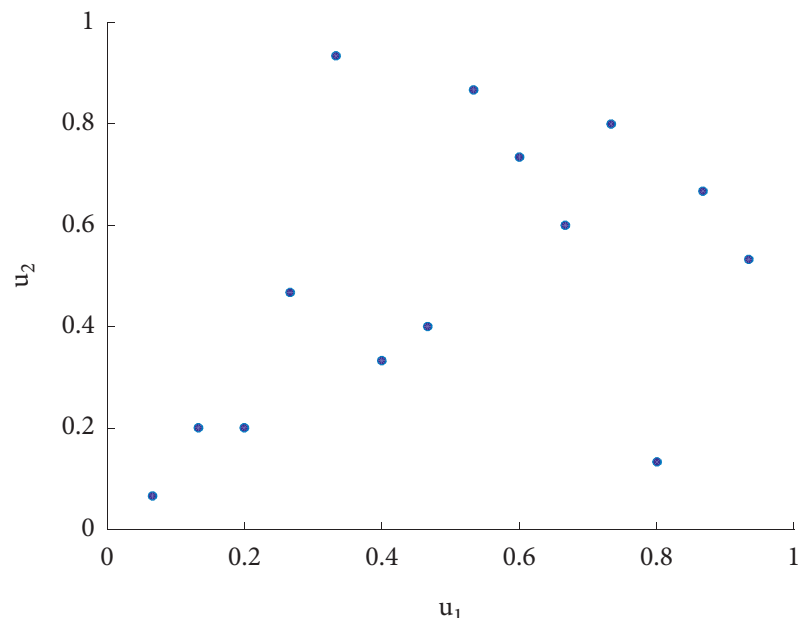

(b)

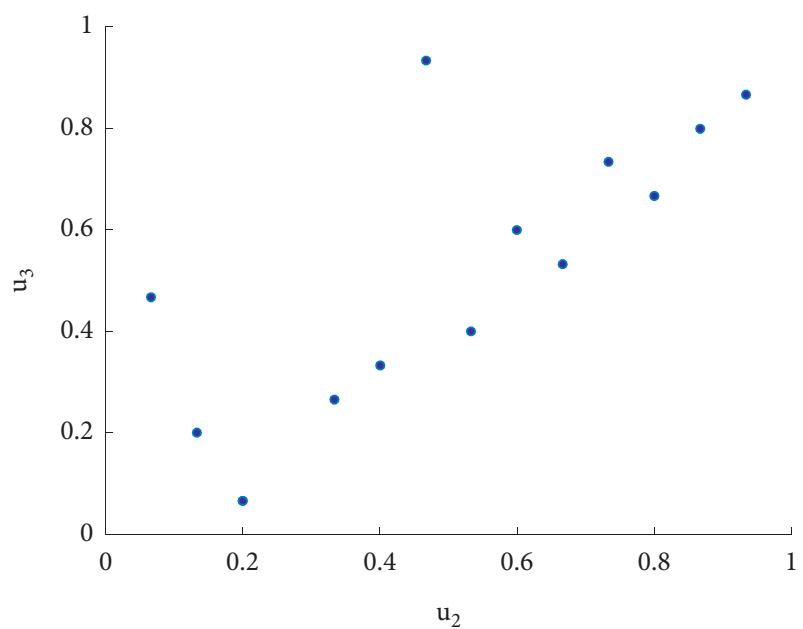

(d)

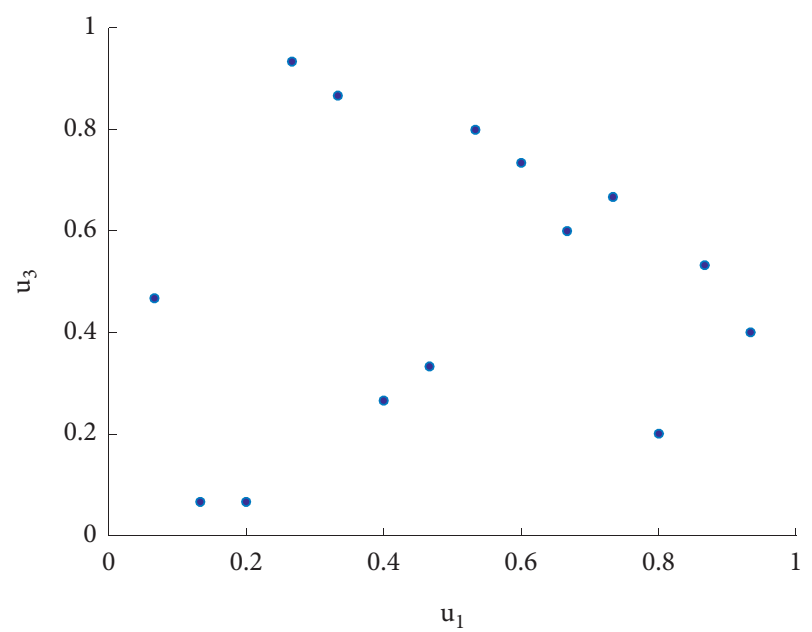

(f)

Figure 1: Scatter plot of (a) $D$ and $Y L$ in the original space, (b) $D$ and $Y L$ in the unit square space, (c) $Y L$ and $E L$ in the original space, (d) $Y L$ and $E L$ in the unit square space, (e) $D$ and $E L$ in the original space, and (f) $D$ and $E L$ in the unit square space. 


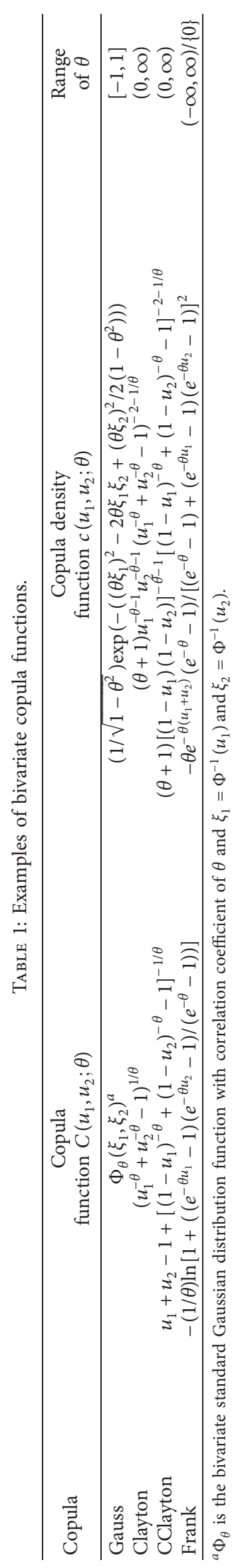


TABle 2: Copula parameters and AIC and BIC values for the Gaussian, Clayton, CClayton, and Frank copulas.

\begin{tabular}{lcccc}
\hline Variables & Gaussian $[\theta$, AIC, BIC] & Clayton $[\theta$, AIC, BIC] & CClayton $[\theta$, AIC, BIC] & Frank $[\theta$, AIC, BIC] \\
\hline$D-Y L$ & $0.405,-0.5090,0.1301$ & $1.8155,-\mathbf{5 . 1 2 2 7}, \mathbf{- 4 . 4 8 3 7}$ & $0.4982,1.3634,2.0024$ & $3.3413,-0.8961,-0.2570$ \\
$Y L-E L$ & $0.7028,-7.5255,-6.8864$ & $1.5296,-5.4303,-4.7913$ & $2.1182,-7.4223,-6.7832$ & $8.1475,-\mathbf{9 . 9 6 0 6 , - 9 . 3 2 1 6}$ \\
$D-E L$ & $0.1422,1.7147,2.3538$ & $0.5598, \mathbf{0 . 4 4 5 9}, \mathbf{1 . 0 8 4 9}$ & $1.4509 e-6,2.0000,2.6391$ & $0.8791,1.7580,2.3970$ \\
\hline
\end{tabular}

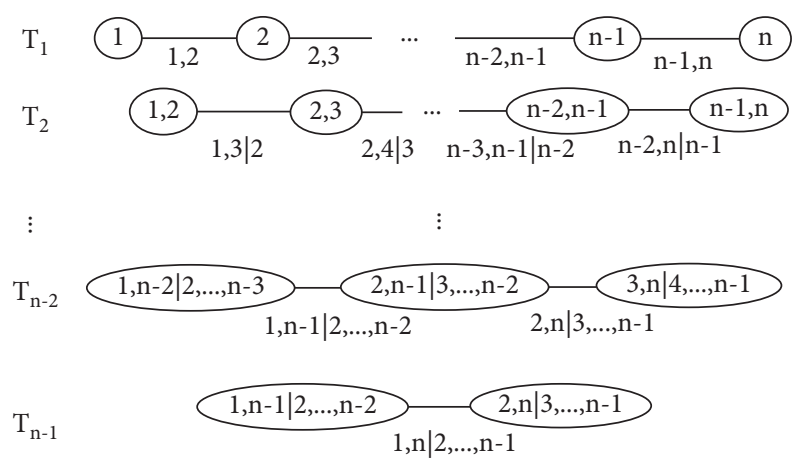

(a)

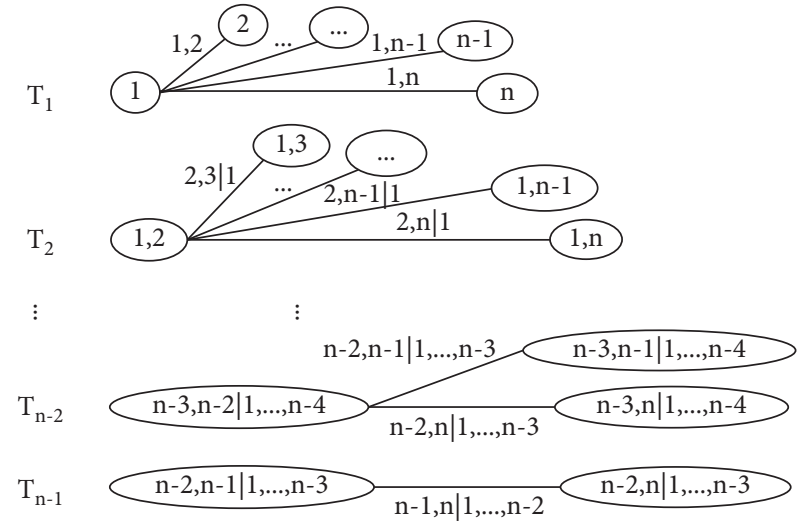

(b)

FIgURE 2: Pair-copula constructions: (a) a D-vine and (b) a C-vine, with $n(n>3)$ variables, $n-1$ trees, and $n(n-1) / 2$ edges.

$$
\begin{aligned}
& f_{\mathrm{X}}(\mathrm{X})=\prod_{k=1}^{n} f_{k}\left(x_{k}\right) \prod_{j=1}^{n-1} \prod_{i=1}^{n-j} c_{i, i+j \mid i+1, \ldots, i+j-1}\left\{F\left(x_{i} \mid x_{i+1}, \ldots, x_{i+j-1}\right), F\left(x_{i+j} \mid x_{i+1}, \ldots, x_{i+j-1}\right)\right\}, \\
& f_{\mathrm{X}}(\mathrm{X})=\prod_{k=1}^{n} f_{k}\left(x_{k}\right) \prod_{j=1}^{n-1} \prod_{i=1}^{n-j} c_{j, j+i \mid 1, \ldots, j-1}\left\{F\left(x_{j} \mid x_{1}, \ldots, x_{j-1}\right), F\left(x_{j+i} \mid x_{1}, \ldots, x_{j-1}\right)\right\} .
\end{aligned}
$$

3.2. Retrieving Vine Copula Parameters from Incomplete Probability Information. The determination of the paircopula parameters is critical to the modeling of multiple dependences. Given the marginal distributions and correlations, the copula parameter can be retrieved using the following relationship [28]:

$$
\rho_{i j}=\int_{-\infty}^{+\infty} \int_{-\infty}^{+\infty} \frac{\left(x_{i}-\mu_{i}\right)}{\sigma_{i}} \frac{\left(x_{j}-\mu_{j}\right)}{\sigma_{j}} f\left(x_{i}, x_{j}\right) \mathrm{d} x_{i} \mathrm{~d} x_{j},
$$

where $\mu_{i}\left(\mu_{j}\right)$ and $\sigma_{i}\left(\sigma_{j}\right)$ are the means and the standard deviations of $x_{i}\left(x_{j}\right)$, respectively, and $f\left(x_{i}, x_{j}\right)$ is the bivariate joint PDF, which can be obtained from the multivariate joint PDF by

$$
f\left(x_{i}, x_{j}\right)=\frac{f_{\mathrm{X}}(\mathrm{X})}{f\left(\mathrm{X}_{-i,-j} \mid x_{i}, x_{j}\right)}
$$

where $\mathrm{X}_{-i,-j}$ denotes the random vector excluding the $i$ th and $j$ th variables. By analogy with equation (6), the conditional PDF $f\left(\mathrm{X}_{-i,-j} \mid x_{i}, x_{j}\right)$ can also be written as a product of marginal conditional PDFs. For example,

$$
f\left(\mathrm{X}_{-i,-j} \mid x_{i}, x_{j}\right)=f\left(x_{1} \mid x_{i}, x_{j}\right) f\left(x_{2} \mid x_{1}, x_{i}, x_{j}\right) \ldots f\left(x_{n} \mid \mathrm{X}_{-i,-j,-n}, x_{i}, x_{j}\right) .
$$

In particular, for a bivariate distribution $f\left(x_{1}, x_{2}\right)$, there is only one unconditional bivariate copula function involved, and its parameter can be solely determined by

$$
\rho_{12}=\int_{-\infty}^{+\infty} \int_{-\infty}^{+\infty} \frac{\left(x_{1}-\mu_{1}\right)}{\sigma_{1}} \frac{\left(x_{2}-\mu_{2}\right)}{\sigma_{2}} f_{1}\left(x_{1}\right) f_{2}\left(x_{2}\right) c_{1,2}\left(F_{1}\left(x_{1}\right), F_{2}\left(x_{2}\right)\right) \mathrm{d} x_{1} \mathrm{~d} x_{2} .
$$


If $f_{\mathrm{X}}(\mathrm{X})$ is a multivariate distribution, conditional copulas must be involved in the decomposition. However, the associated conditional copula parameters cannot be analytically solved from one single integral function. For example, suppose a trivariate distribution $f\left(x_{1}, x_{2}, x_{3}\right)$, which can be represented by three copulas: $c_{1,2}\left(F_{1}\left(x_{1}\right), F_{2}\left(x_{2}\right)\right), \quad c_{2,3}\left(F\left(x_{2}\right), F\left(x_{3}\right)\right), \quad$ and $c_{1,3 \mid 2}\left(F\left(x_{1} \mid x_{2}\right), F\left(x_{3} \mid x_{2}\right)\right)$, and three marginal PDFs. To relate the conditional copula parameter $\theta_{1,3 \mid 2}$ with a correlation coefficient (such as $\rho_{1,3}$ ), the conditional distribution has to be represented by other conditional copulas (e.g., $f\left(x_{2} \mid x_{1}, x_{3}\right)=c_{1,2 \mid 3}\left(F\left(x_{2} \mid x_{3}\right), F\left(x_{1} \mid x_{3}\right)\right) c_{2,3}\left(F\left(x_{2}\right)\right.$, $\left.\left.F\left(x_{3}\right)\right) f_{2}\left(x_{2}\right)\right)$; otherwise, the desired parameter $\theta_{1,3 \mid 2}$ to be estimated will be removed from equation (11) after representing the bidimensional marginal PDF by equation (12). In other words, the analytical approach to retrieving the desired conditional copula parameters requires a simultaneous estimate of irrelevant copula parameters (such as $\theta_{1,2 \mid 3}$ in the above trivariate example). To address the problem, a sequential search strategy (S3) and a simple simulation-based bisection search method proposed in [21,22] were adopted to approximate the pair-copula parameters. The major advantage of the algorithm is that only one copula parameter is estimated per iteration and the estimate of irrelevant parameters is avoided. For stationary stochastic processes, the correlation coefficient is dependent on the relative separation such that the correlation coefficients between any two neighboring nodes in the $\mathrm{D}$-vine structure are identical. Moreover, the marginal distribution of each node is identical if the stochastic process is stationary. Thus, the pair-copula parameters at each tree level are identical, and the efforts for parameter searching can be significantly reduced. For this reason, the $\mathrm{D}$-vine structured model is particularly suitable for reliability problems involving stationary stochastic processes.

\section{Subset Simulation considering Dependent Random Variables}

4.1. Subset Simulation. The Monte Carlo simulation (MCS) is widely applied to estimate the failure probability given in equation (1) due to the simplicity and robustness of the algorithm [29]. However, the crude MCS can be unacceptable for its high computational demands when assessing small failure probabilities [30]. To address the limitation, various advanced simulation techniques have been proposed [31]. The SS uses a Markov Chain Monte Carlo (MCMC) sampling strategy, which is identified as one of the most efficient method for estimating small failure probabilities [32-34]. Let $F$ denote the failure event $F=\left\{\mathrm{U} \in \mathbb{R}^{n}: G(\mathrm{U}) \leq 0\right\}$ in the $U$-space. The basic idea of SS is to progressively approach the target failure region $F$ through a sequence of nested subsets of the random space:

$$
F=F_{m} \subset F_{m-1} \subset \cdots \subset F_{1} \subset F_{0}=\mathbb{R}^{n},
$$

where $F_{0}$ is the certain event. By conditioning on the intermediate failure regions $F_{1}, F_{2}, \ldots, F_{m}$, the failure probability is estimated as a product of conditional probabilities:

$$
P(F)=P\left(F_{1}\right) \prod_{j=2}^{m} P\left(F_{j} \mid F_{j-1}\right) .
$$

The probability $P\left(F_{1}\right)$ is equivalently computed by crude MCS. To estimate the conditional probabilities $P\left(F_{j} \mid F_{j-1}\right), j=2, \ldots, m$, samples from the conditional PDFs $\left\{\varphi_{n}\left(\mathrm{U} \mid F_{j-1}\right): j=2, \ldots, m\right\}$ should be generated, which is

$$
\varphi_{n}\left(\mathrm{U} \mid F_{j-1}\right)=\frac{\varphi_{n}(\mathrm{U}) I_{F_{j-1}}(\mathrm{U})}{P\left(F_{j-1}\right)} .
$$

In equation (17), $I_{F_{j-1}}(\mathrm{U})$ is the indicator function of $F_{j-1}$. Generation of independent and identically distributed (i.i.d.) samples from $\varphi_{n}\left(\mathrm{U} \mid F_{j-1}\right)$ can be achieved by application of the acceptance-rejection method.

Without loss of generality, suppose that the intermediate and target failure regions are defined as $F_{j}=\left\{\mathrm{U} \in \mathbb{R}^{n}: G(\mathrm{U}) \leq c_{j}\right\}$, where $c_{1}>c_{2}>\cdots>c_{m}=0$. The values of $\left\{c_{j}: j=1, \ldots, m-1\right\}$ can be chosen adaptively so that the estimates of the conditional probabilities $P\left(F_{j} \mid F_{j-1}\right), j=1, \ldots, m$, equal to a constant value of $P_{0}$.

For details of the implementation procedure of SS, readers are referred to [32].

4.2. Rosenblatt Transformation. The original SS assumes that the random variables are mutually independent (or have been transformed into independent ones). To incorporate the correlations into reliability evaluation, the iso-probabilistic transformation $\mathrm{U}=T(\mathrm{X})$ that maps the dependent random variables into independent ones is needed [29]. The probability of failure can thus be expressed in the transformed space as

$$
p_{f}=\int_{G\left(T^{-1}(\mathrm{U}) \leq 0\right)} I(\mathrm{U}) \varphi_{n}(\mathrm{U}) \mathrm{dU},
$$

where $\varphi_{n}(\mathrm{U})=\prod_{i=1}^{n} \varphi\left(U_{i}\right), \varphi(\cdot)$ is the standard normal PDF, and $G(\mathrm{X})=G\left(T^{-1}(\mathrm{U})\right)$ is the limit-state function in the independent standard normal space ( $U$-space), and $I(\mathrm{U})$ is the indicator function, where $I(\mathrm{U})=1$ if $G(\mathrm{U}) \leq 0$ and $I(\mathrm{U})=0$, otherwise.

The Rosenblatt transformation can be used for simulating nonlinear dependent random variables [35]. The Rosenblatt transformation $T: \mathbb{R}^{n} \longrightarrow \mathbb{R}^{n}$ is defined as

$$
\begin{aligned}
\mathrm{U} & =T(\mathrm{X}) \\
& =\left(\begin{array}{c}
\Phi^{-1}\left(z_{1}\right) \\
\Phi^{-1}\left(z_{2}\right) \\
\vdots \\
\Phi^{-1}\left(z_{n}\right)
\end{array}\right) \\
& =\left(\begin{array}{c}
\Phi^{-1}\left(F\left(x_{1}\right)\right) \\
\Phi^{-1}\left(F\left(x_{2} \mid x_{1}\right)\right) \\
\vdots \\
\Phi^{-1}\left(F\left(x_{n} \mid x_{1}, \ldots, x_{n-1}\right)\right)
\end{array}\right),
\end{aligned}
$$


where $\Phi^{-1}$ is the inverse function of the CDF of a univariate standard Gaussian distribution and $F\left(x_{n} \mid x_{1}, \ldots, x_{n-1}\right)$ is the marginal conditional distributions. By using non-Gaussian copulas, the nonlinearity of dependency is expressed in the copula representation of the marginal conditional distributions $F\left(x_{n} \mid x_{1}, \ldots, x_{n-1}\right)$. For simplicity, the excluding variable $v_{j}$ in the $\mathrm{C}$-vine model is always chosen so that

$$
F\left(x_{j} \mid x_{1}, \ldots, x_{j-1}\right)=h_{j \mid j-1: 1, \ldots, j-2}\left(F\left(x_{j} \mid x_{1}, \ldots, x_{j-2}\right), F\left(x_{j-1} \mid x_{1}, \ldots, x_{j-2}\right)\right)
$$

and $v_{j}$ of the D-vine models is chosen so that

$$
F\left(x_{j} \mid x_{1}, \ldots, x_{j-1}\right)=h_{j \mid 1: 2, \ldots, j-1}\left(F\left(x_{j} \mid x_{2}, \ldots, x_{j-1}\right), F\left(x_{1} \mid x_{2}, \ldots, x_{j-1}\right)\right) .
$$

Then, the dependent random variables in the original space can be obtained from independent random variables using the inverse of the Rosenblatt transformation. In particular, for C-vine models,

$$
x_{k}=F_{k}^{-1}\left(h_{k \mid 1}^{-1}\left(h_{k \mid 2: 1}^{-1}\left(\ldots h_{k \mid k-1: 1,2, \ldots, k-2}^{-1}\left(\Phi\left(U_{k}\right), F_{k-1}\left(x_{k-1}\right)\right) \ldots, F_{2}\left(x_{2}\right)\right), F_{1}\left(x_{1}\right)\right)\right)
$$

and for D-vine models,

$$
x_{k}=F_{k}^{-1}\left(h_{k \mid k-1}^{-1}\left(h_{k \mid k-2: k-1}^{-1}\left(\ldots h_{k \mid 1: 1,2, \ldots, k-1}^{-1}\left(\Phi\left(U_{k}\right), F\left(x_{1} \mid x_{2}, \ldots, x_{k-1}\right)\right) \ldots, F\left(x_{k-2} \mid x_{k-1}\right)\right), F\left(x_{k-1}\right)\right)\right) \text {, }
$$

where $h^{-1}(\cdot)$ is the inverse function of equation (8).

\section{Numerical Examples}

In this section, two examples of FE analysis are used to demonstrate the effect of nonlinear dependency on structural reliability. For both examples, the sample size at each SS simulation level is set to 10000 and the conditional failure probability $p_{0}$ is set to 0.1 . The aim of using a relatively larger sample size is to provide a more robust estimate. A uniform proposal PDF is used for MCMC simulation. The spread of the uniform PDF is taken as twice of the standard deviation of each random variable at the current level.

\subsection{Example 1: Three-Bay Five-Story Frame Structure.} The three-bay five-story frame structure has been analyzed in many studies [36-38]. The schematic diagram of the structure is shown in Figure 3. There are 21 random variables representing various loads and material properties. The 21 random variables are independent by three blocks. The first block involves 3 correlated random variables representing external loads $\left(P_{1}, P_{2}\right.$, and $P_{3}$ with pairwise correlation coefficients of 0.5 ). The second block involves 2 correlated random variables representing the modulus of beams and columns, respectively ( $E_{4}$ and $E_{5}$ with a correlation coefficient of 0.9 ). The third block involves the other 16 correlated random variables representing material properties including cross-sectional area $A$ and moment of inertia $I$. The cross- sectional area and the moment of inertia of the same element are highly correlated with a correlation coefficient of 0.95 while those of different elements are assumed a correlation coefficient of 0.13 (i.e., $\rho_{A_{i} I_{i}}=0.95$ and $\rho_{A_{i} A_{j}}=\rho_{I_{i} I_{j}}$ $\left.=\rho_{I_{j} A_{j}}=0.13\right)$. The element properties and their statistics are listed in Tables 3 and 4, respectively.

The structure is considered failure if the horizontal displacement of the upper floor exceeds a threshold value of $0.061 \mathrm{~m}$. Thus, the limit state function is

$$
G(\mathrm{X})=0.061-\Delta x .
$$

The bivariate Gaussian copula is first adopted for all pair copulas. The result corresponding to this linear dependency should be consistent with that based on the Nataf transformation [12]. Table 5 compares the failure probability by the extended SS with results in previous works. The consistent results indicate the accuracy of the proposed method under linear dependency assumption.

To investigate the impact of nonlinear dependency on the structural reliability, the correlated loads are assumed to have an upper tail correlation that can be captured by a Clayton copula, while the correlated material properties are assumed to have a lower tail correlation that is better modeled by a Clayton copula. The failure probability under this hybrid copulas is $p_{f}=8.3749 \times 10^{-4}$, which is $51 \%$ higher than the Gaussian copula case. This deviation may seem not significant compared to that caused by other uncertainties. However, the difference can enlarge as the 


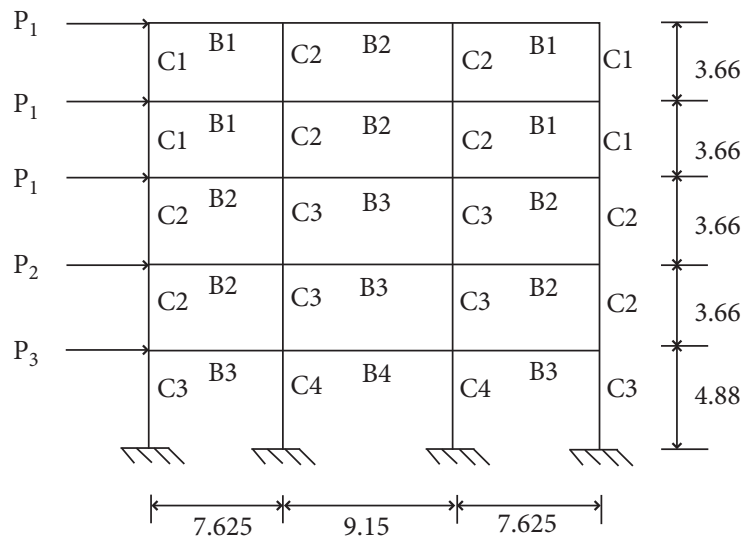

FIGURE 3: Frame structure and its elements (unit: m).

Table 3: Frame element properties for example 1.

\begin{tabular}{lccc}
\hline Element & Modulus of elasticity & Moment of inertia & Cross section \\
\hline$B 1$ & $E_{4}$ & $I_{10}$ & $A_{10}$ \\
$B 2$ & $E_{4}$ & $I_{11}$ & $A_{11}$ \\
$B 3$ & $E_{4}$ & $I_{12}$ & $A_{12}$ \\
$B 4$ & $E_{4}$ & $I_{13}$ & $A_{13}$ \\
$C 1$ & $E_{5}$ & $I_{6}$ & $A_{6}$ \\
$C 2$ & $E_{5}$ & $A_{7}$ \\
$C 3$ & $E_{5}$ & $I_{8}$ & $A_{8}$ \\
$C 4$ & $E_{5}$ & $I_{9}$ & $A_{9}$ \\
\hline
\end{tabular}

TABle 4: Statistics of element properties for example 1.

\begin{tabular}{lcccc}
\hline Variable & Distribution & Unit & Mean value & Standard deviation \\
\hline$P_{1}$ & Gumbel max & $\mathrm{kN}$ & 133.454 & 40.04 \\
$P_{2}$ & Gumbel max & $\mathrm{kN}$ & 88.97 & 35.59 \\
$P_{3}$ & Gumbel max & $\mathrm{kN}$ & 71.175 & 28.47 \\
$E_{4}$ & Normal & $\mathrm{kN} / \mathrm{m}^{2}$ & $2.173752 \times 10^{7}$ & $1.9152 \times 10^{6}$ \\
$E_{5}$ & Normal & $\mathrm{kN} / \mathrm{m}^{2}$ & $2.379636 \times 10^{7}$ & $1.9152 \times 10^{6}$ \\
$I_{6}$ & Normal & $\mathrm{m}^{4}$ & $0.813443 \times 10^{-2}$ & $1.08344 \times 10^{-3}$ \\
$I_{7}$ & Normal & $\mathrm{m}^{4}$ & $1.150936 \times 10^{-2}$ & $1.298048 \times 10^{-3}$ \\
$I_{8}$ & Normal & $\mathrm{m}^{4}$ & $2.137452 \times 10^{-2}$ & $2.59609 \times 10^{-3}$ \\
$I_{9}$ & $\mathrm{~m}^{4}$ & $2.596095 \times 10^{-2}$ & $3.028778 \times 10^{-3}$ \\
$I_{10}$ & $\mathrm{~m}^{4}$ & $1.081076 \times 10^{-2}$ & $2.596095 \times 10^{-3}$ \\
$I_{11}$ & Normal & $\mathrm{m}^{4}$ & $1.410545 \times 10^{-2}$ & $3.46146 \times 10^{-3}$ \\
$I_{12}$ & Normal & $\mathrm{m}^{4}$ & $2.327853 \times 10^{-2}$ & $5.624873 \times 10^{-3}$ \\
$I_{13}$ & $\mathrm{~m}^{4}$ & $2.596095 \times 10^{-2}$ & $6.490238 \times 10^{-3}$ \\
$A_{6}$ & Normal & $\mathrm{m}^{2}$ & 0.312564 & 0.055815 \\
$A_{7}$ & Normal & $\mathrm{m}^{2}$ & 0.3721 & 0.07442 \\
$A_{8}$ & Normal & $\mathrm{m}^{2}$ & 0.50606 & 0.093025 \\
$A_{9}$ & Normal & $\mathrm{m}^{2}$ & 0.55815 & 0.11163 \\
$A_{10}$ & Normal & $\mathrm{m}^{2}$ & 0.253028 & 0.10230225 \\
$A_{11}$ & Normal & $\mathrm{m}^{2}$ & 0.29116825 & 0.1209325 \\
$A_{12}$ & Normal & $\mathrm{m}^{2}$ & 0.37303 & 0.195375 \\
$A_{13}$ & Normal & & & \\
\hline & Normal & Normal & &
\end{tabular}

TABLE 5: Example 1: comparison of reliability results under linear dependencies.

\begin{tabular}{lcccr}
\hline Method & This study & {$[36]$} & {$[37]$} & [38] \\
\hline$\beta$ & 3.2612 & 3.29 & 3.220 & 3.218 \\
$P_{f}\left(\times 10^{-3}\right)$ & 5.5466 & 5.009 & 6.410 & 6.454 \\
\hline
\end{tabular}


expected failure probability decreases. As shown in Figure 4, $\eta$ denotes the ratio of the failure probability under the nonlinear dependency over that of the linear case, which increased as the tolerant displacement $\varepsilon$ becomes larger. Note that structural systems are often designed towards high reliability, which implies that the effect of dependency can be significant.

To illustrate how reliability results are biased by nonlinear dependency, Figure 5 presents the MCMC simulation in successively generating one pair of correlated random variables $\left(E_{4}\right.$ and $\left.E_{5}\right)$ under different copulas. With the use of a fixed seed, the random walks under different dependencies are exactly the same in the $U$-space. However, the values differed after mapping into the original space despite a similar route is observed. This effect is also true for multiple nonlinear dependencies. The reason is that the nonlinear characteristics for each pair of uncertain parameters can be maintained using the same non-Gaussian copula in a vine structure regardless of their conditioning order [39]. For example, compared to the Gaussian copula, the cross-sectional area and moment of inertia are more likely to have similar values if any one of these parameters has a small value under the Clayton copula.

\subsection{Example 2: The Cantilever Beam with Spatially Variable} Modulus. The cantilever beam has been analyzed by several researchers $[40,41]$. Consider a cantilever beam with a length of $L=1$ unit and a constant cross-sectional area $A=1$ unit, as shown in Figure 6 . The beam is subjected to a linearly increasing uniaxial load $P(x)=x$ along the $x$-direction. The modulus of elasticity varies spatially along the $x$-direction that

$$
E(x)=\mu_{E}[1+\alpha(x)]
$$

where $\mu_{E}=1$ unit is the mean of elastic modulus and $\alpha(x)$ is a homogeneous Gaussian random field with mean zero and autocovariance function given by

$$
\begin{aligned}
\Gamma_{\alpha}(\xi) & =\operatorname{Ex}[\alpha(x) \alpha(x+\xi)] \\
& =\sigma_{E}^{2} \exp \left(-\frac{|\xi|}{b L}\right),
\end{aligned}
$$

where $x$ and $x+\xi$ are the coordinates of two sections of the beam, $E x[\cdot]$ is the expectation operator, $\sigma_{E}=0.1$ is the standard deviation of $\alpha(x)$, and $b=100$ is the correlation length. Although the proposed method is illustrated with a Gaussian process, the application to non-Gaussian processes is straightforward since the choice of marginal distribution is independent.

Two different meshes were used for the discretization of the FE model and the random field, respectively. To provide a consistent comparison with previous works, the random field is discretized into 256 random variables and further averaged every 16 points. The resulting 16 values of material modulus are then mapped onto the $480 \mathrm{FE}$ meshes. The FE mesh grid is sufficiently fine as the deterministic result converges. The limit state function is defined with respect to the axial displacement at the free end (point B):

$$
G(X)=0.45-\Delta x
$$

Table 6 compares the result with that from previous works, where the random field $\alpha(x)$ was parameterized using the spectral decomposition method (i.e., $\alpha(x)$ ) is represented by a set of independent standard normal random variables $U_{i}$ as $\alpha(x)=\mu_{\alpha(x)}+\sum_{i=1}^{\infty} \sqrt{\lambda_{i}} \psi_{i}(x) U_{i}$, where $\left\{\lambda_{i}, \psi_{i}(x)\right\}$ are the eigenvalue and eigenfunction of the covariance kernel $\operatorname{Cov}\left(x, x^{\prime}\right)$ satisfying $\lambda_{i} \psi_{i}(x)=\int_{\Omega} \operatorname{Cov}(x$, $\left.\left.x^{\prime}\right) \psi_{i}\left(x^{\prime}\right) \mathrm{d} x^{\prime}, x \in \Omega\right)$ [42]. The consistency in failure probability indicates that the proposed method is accurate. The slight deviation can be attributed to the variation of the SS estimator. It has been acknowledged that the spectral decomposition is exact if the random variables within a field are jointly Gaussian [43]. From the copula perspective, jointly Gaussian means Gaussian marginals plus Gaussian copulas [13], which implies a linear dependency is used. However, nonlinear dependencies of stochastic processes have been reported in the literature [15]. Therefore, the effect of nonlinear dependency in a random field is of interest.

To illustrate the impact of nonlinear dependency in a random field, the bivariate Clayton copula and the bivariate Frank copula are, respectively, taken as the pair copulas. Moreover, the effect at different correlation lengths is examined. Figure 7 shows the comparison of failure probabilities as a function of the correlation length. When $b$ approaches to infinity or tends to be zero, the deviation is negligible. This result can be expected as the random variables which are equivalent to a single random variable when fully correlated $(b=\infty)$, while the zero correlations $(b=0)$ lead to the degeneration of all pair copulas to independence copula; thus, the joint probability distribution of the random field is simply a multiplication of its marginals. However, the random field is seldom highly ragged or smooth. For example, the ratio of correlation length over field length $b / L$ is in the order of $10^{-1}$ for various properties of CFRP composite panels [44]. Refer to Figure 7; the failure probability from the linear dependency case can be increased or reduced by more than thousands of times if the dependency is nonlinear. Figure 8 presents the CDFs corresponding to different copulas when $b=100$ and $b=0.1$, respectively. For highly smooth random field $(b=100)$, the deviation is relatively insignificant regardless of the expected failure probability; however, the deviation becomes more significant as the cantilever beam approaches to high reliability when the spatial variation is moderate $(b=0.1)$. Figure 9 shows the realizations of the stochastic process under different dependencies. Note the seed for simulation is identical. The value of modulus at each point is different despite the similar pattern of variance.

Figures $10(\mathrm{a})-10(\mathrm{~d})$ show the means of modulus at different sections as the simulation level increases from 1 to 4 when different copulas are used. The mean values are generally the same at level 1 (crude MCS). The coordinate corresponding to the minimum mean value of modulus, which indicates the probable failure section, shifted to the 


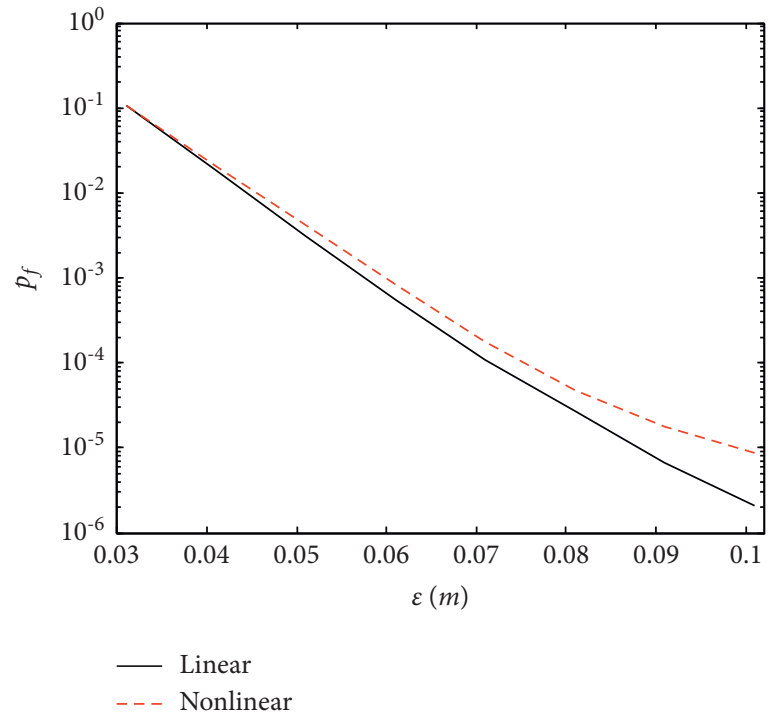

(a)

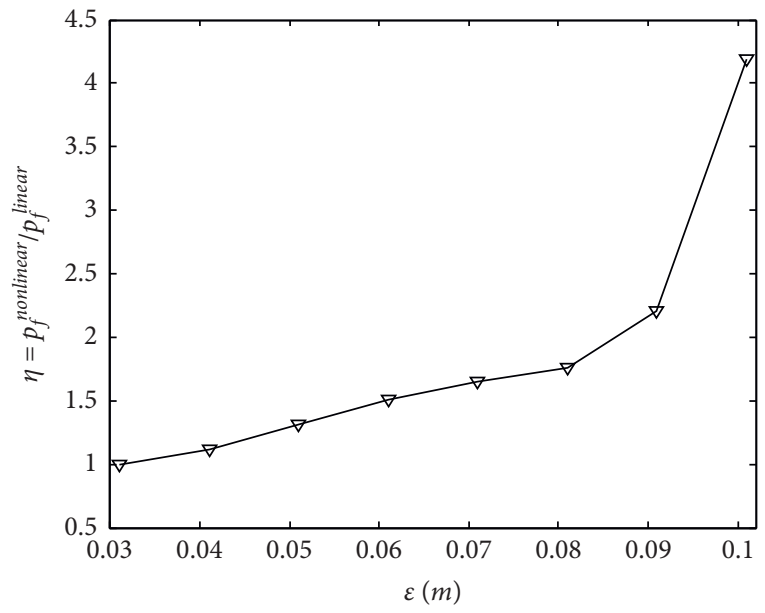

Figure 4: (a) The failure probability under both linear and nonlinear dependencies decreases as the threshold value increases; (b) deviation in failure probabilities at different threshold values.

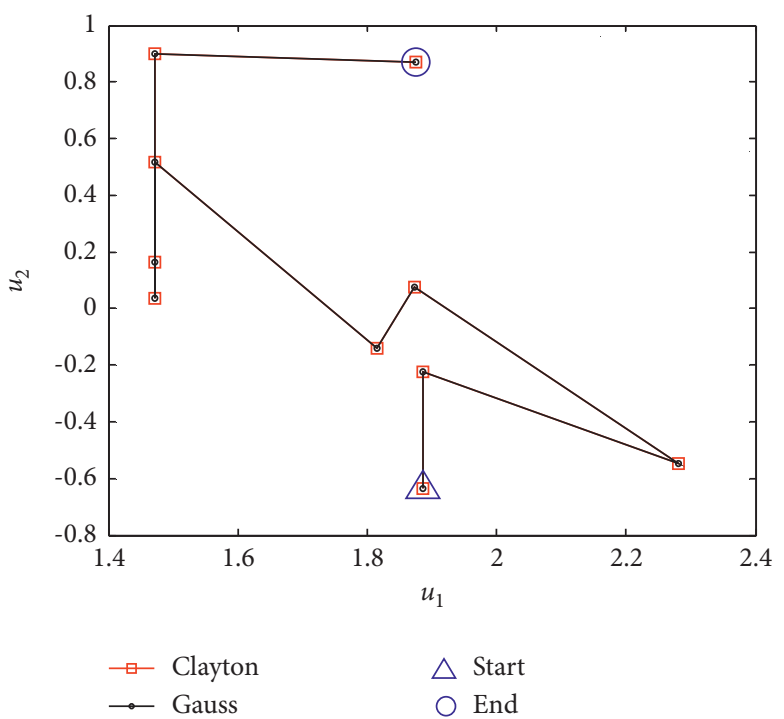

(a)

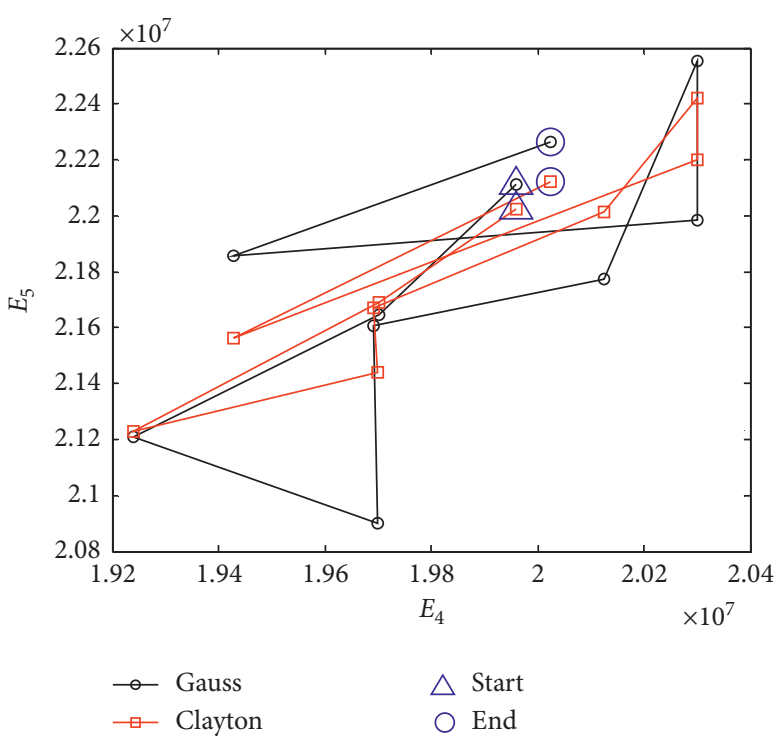

(b)

Figure 5: A single sample after 9 random walks: (a) in the $U$-space and (b) in the original space.

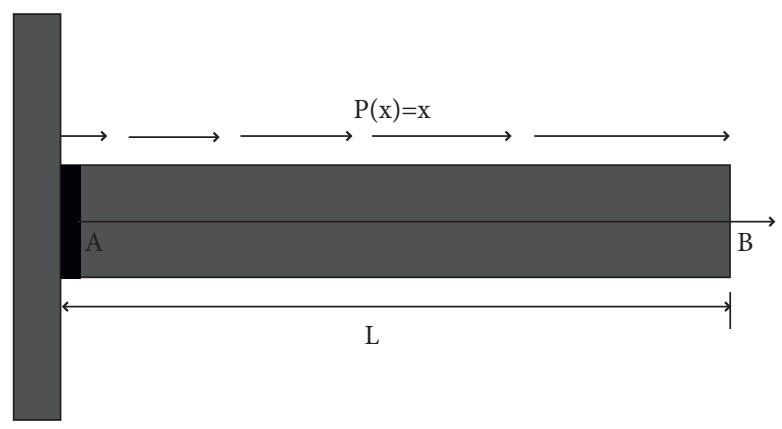

FIGURE 6: Geometry and loads of the beam subjected to a linearly increasing uniaxial load. 
TABLE 6: Comparison of reliability results: example 2.

\begin{tabular}{lccc}
\hline Method & This study & {$[40]$} & {$[41]$} \\
\hline$\beta$ & 2.5556 & 2.5663 & 2.5616 \\
$P_{f}\left(\times 10^{-3}\right)$ & 5.30 & 5.14 & 5.21 \\
\hline
\end{tabular}

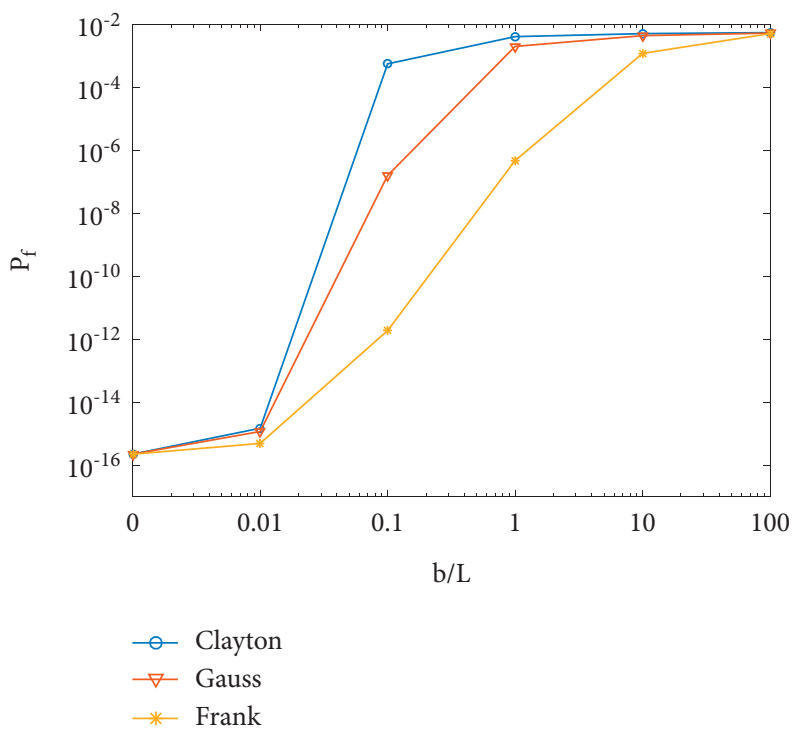

FigURE 7: Effect of dependence structure on failure probability as a function of correlation length.

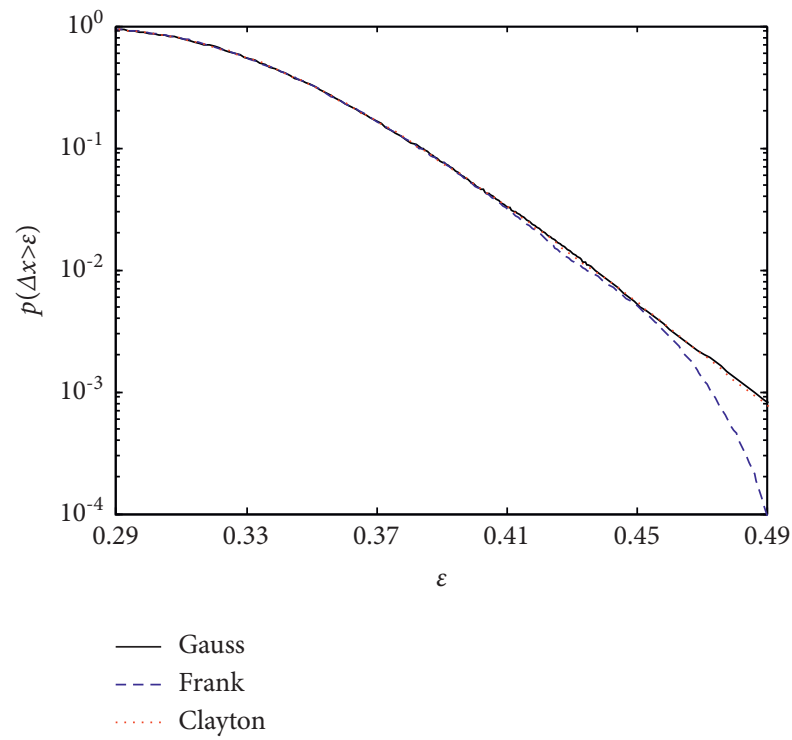

(a)

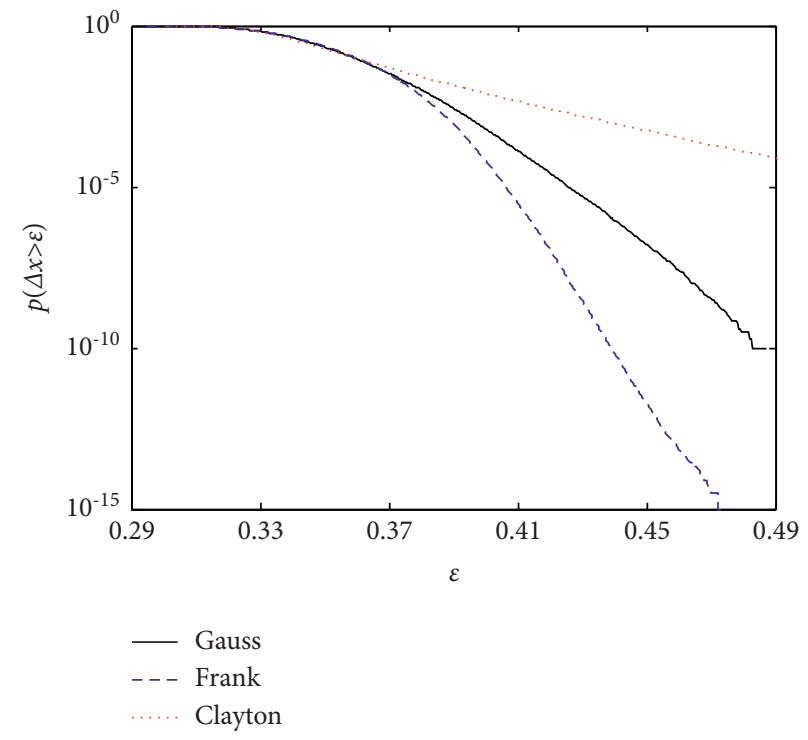

(b)

Figure 8: CDFs of the axial displacement of the cantilever beam under different copulas: (a) $b=100$; (b) $b=0.1$.

left side far away from the free end as the simulation level increased. However, the dependency can influence the minimum mean value at each simulation level. For example, the Clayton copula, which has a lower tail dependency of modulus, makes the targeted failure region reached fast as the minimum mean value of conditional samples decreases sharply. Figures 11-12 further show the PDFs of conditional samples at significant and insignificant section, respectively. 


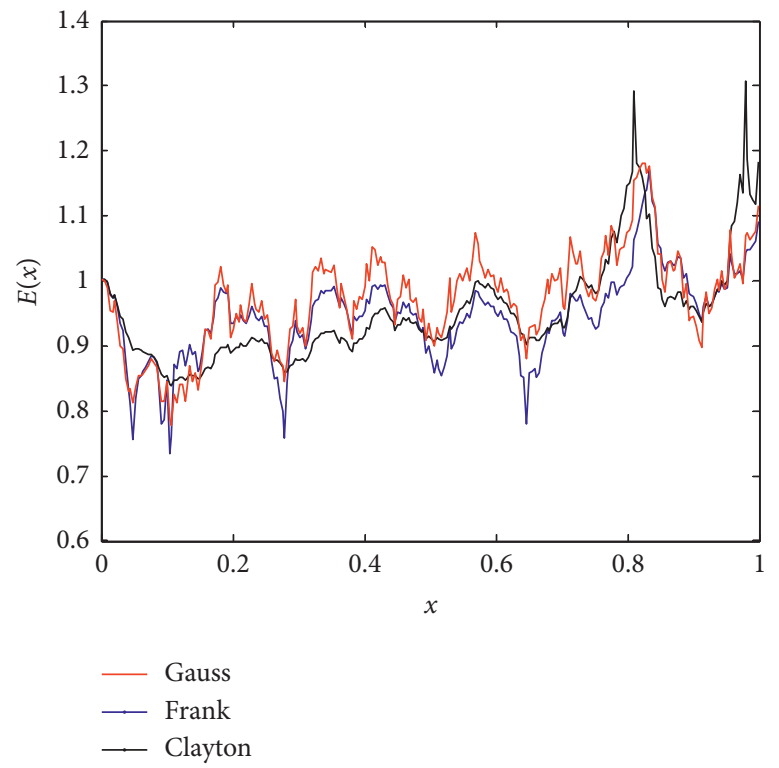

FIGURE 9: Realizations of the spatial variance of modulus under different dependencies.

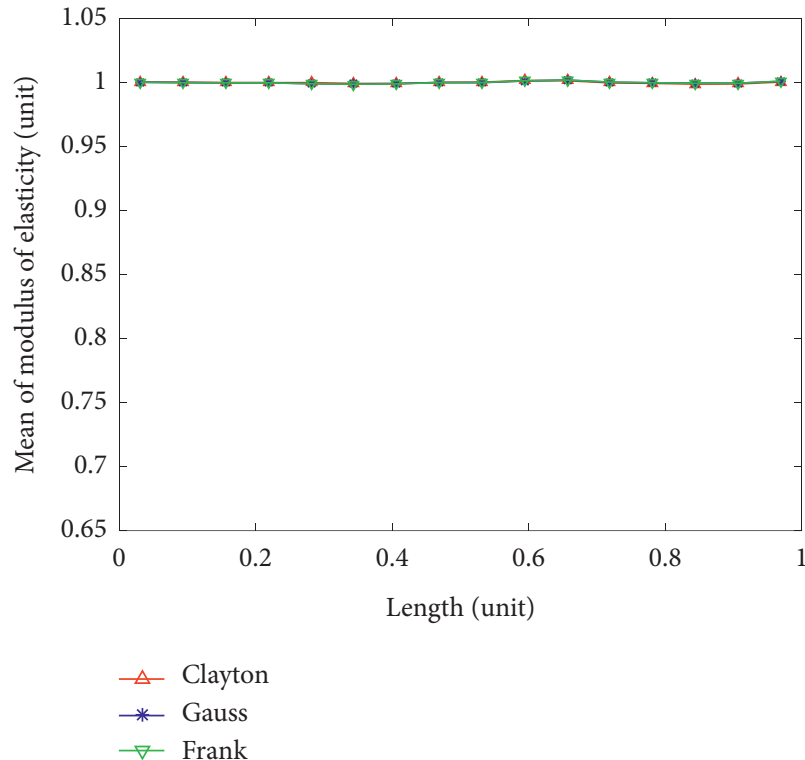

(a)

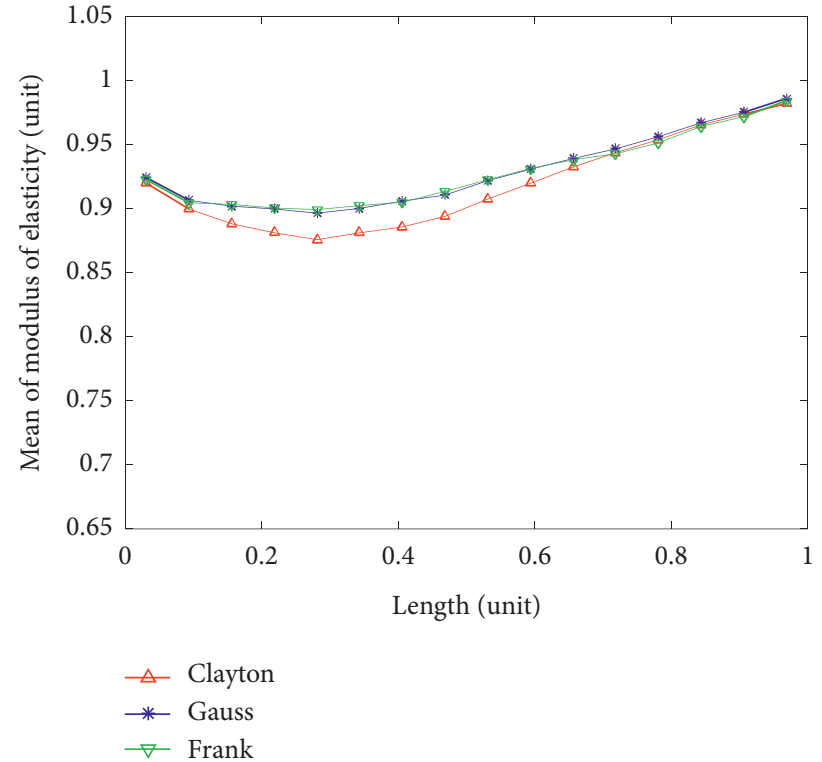

(b)

Figure 10: Continued. 


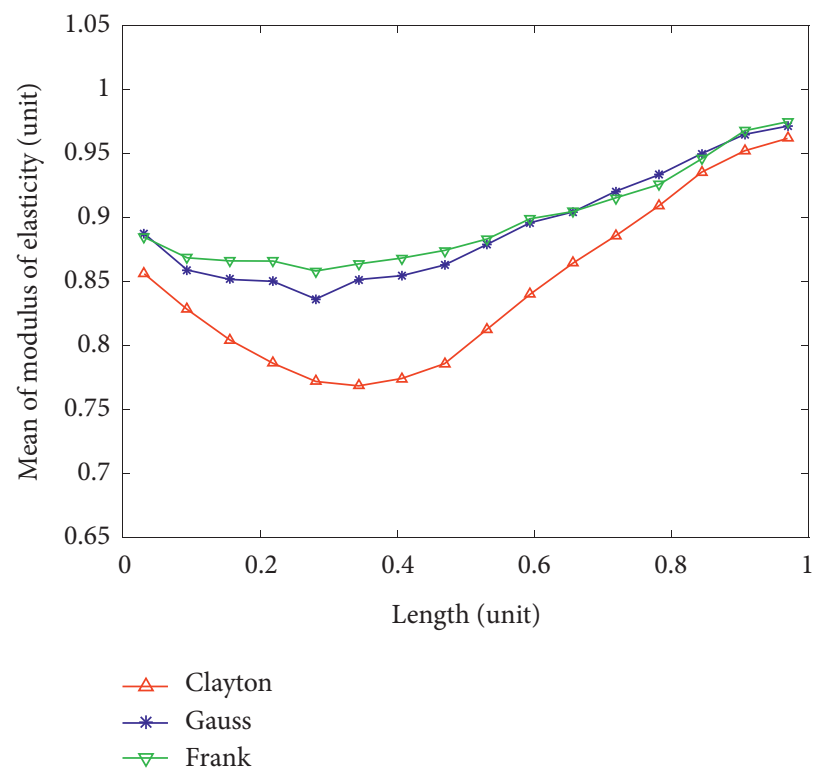

(c)

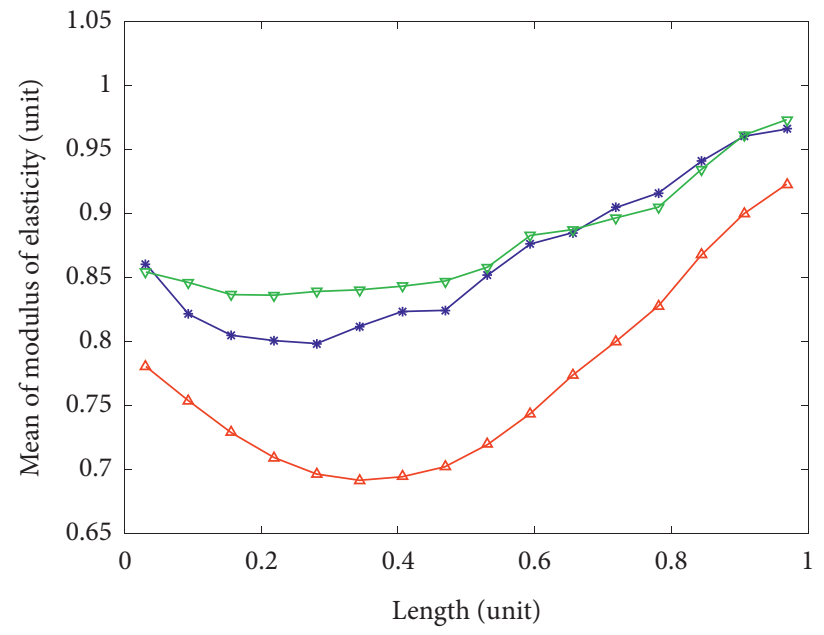

$$
\begin{aligned}
& \triangle \text { Clayton } \\
& * \text { Gauss } \\
& \nabla \text { Frank }
\end{aligned}
$$

(d)

Figure 10: Mean values of $\mathbf{E}$ in different lengths with different dependence structures: (a) at Level 1; (b) at Level 2; (c) at Level 3; (d) at Level 4. 
Level
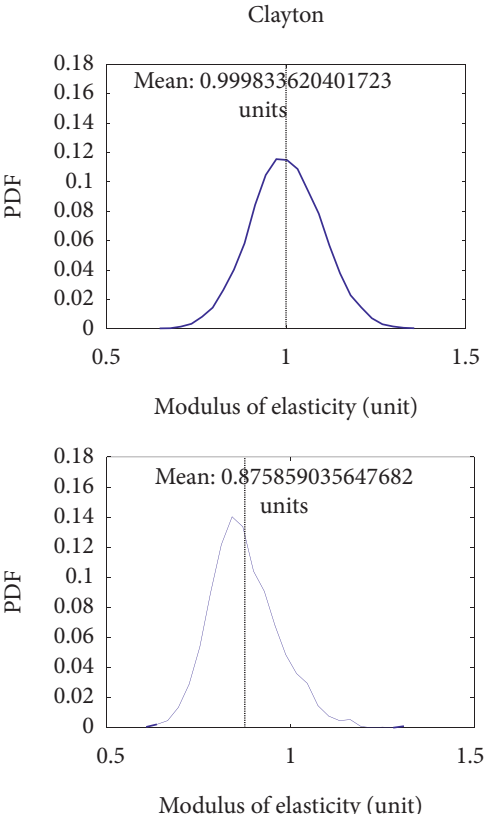

3
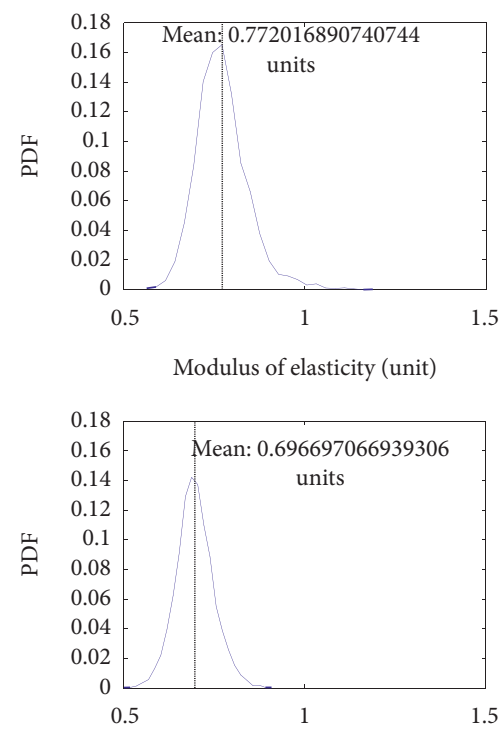

Modulus of elasticity (unit)
Gauss

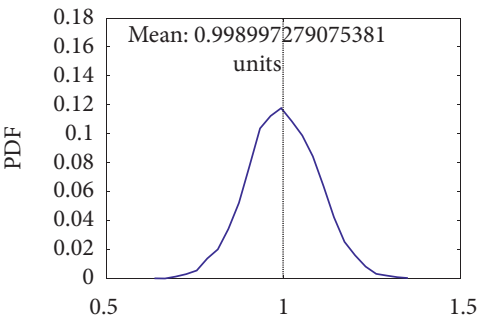

Modulus of elasticity (unit)
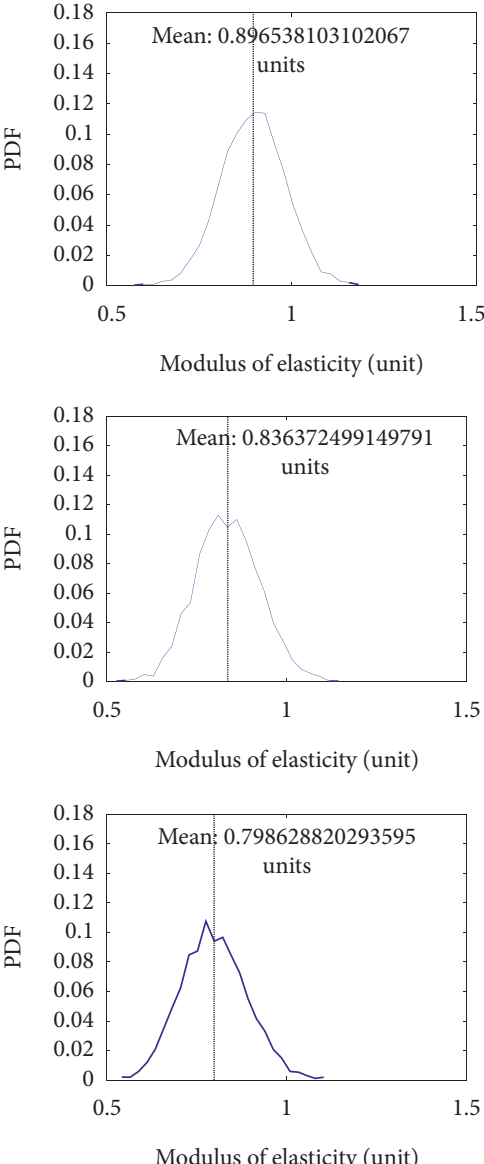

Frank

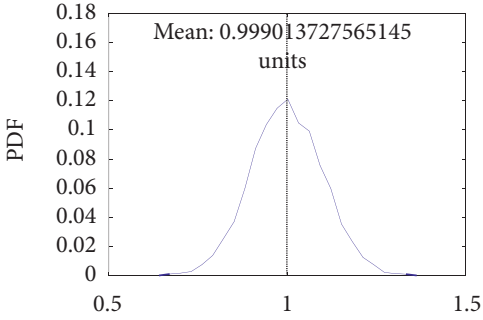

Modulus of elasticity (unit)
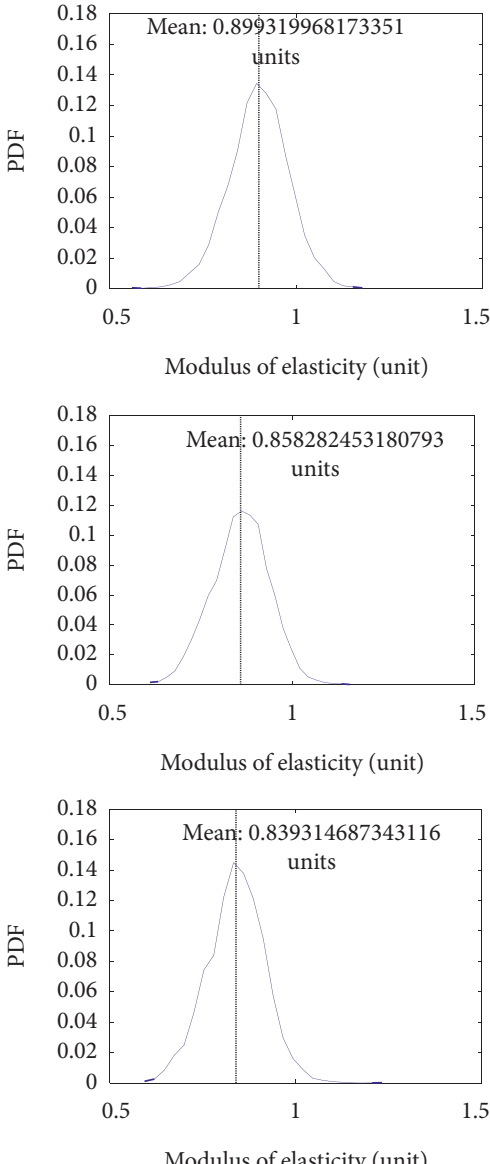

Figure 11: Distribution of modulus of elasticity at significant section: 0.3125 unit. 
Level
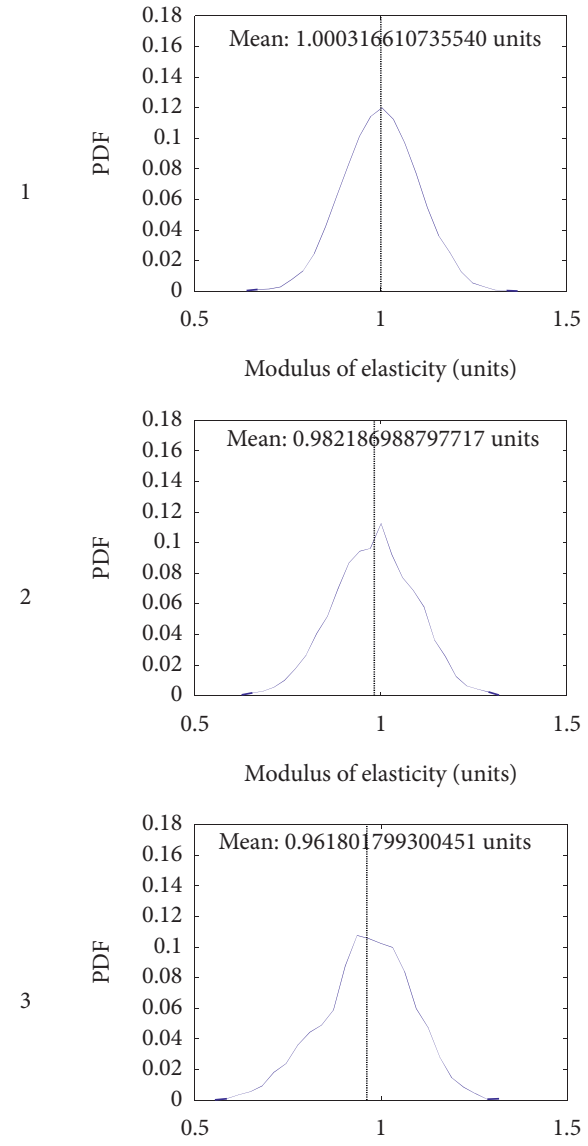

Modulus of elasticity (units)

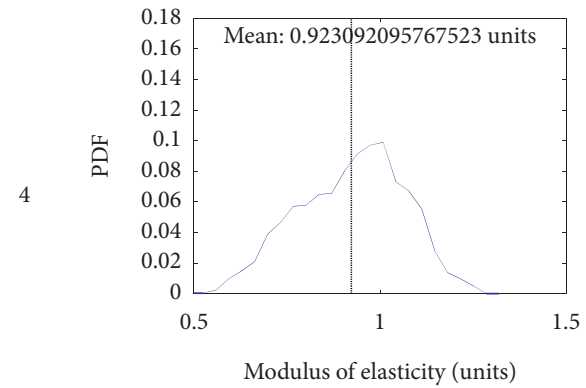

Gauss
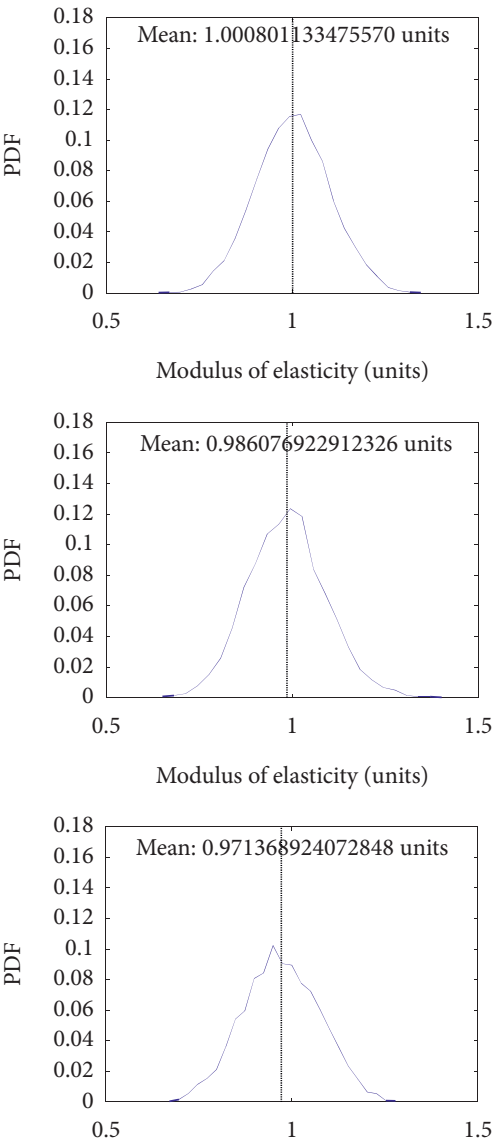

Modulus of elasticity (units)

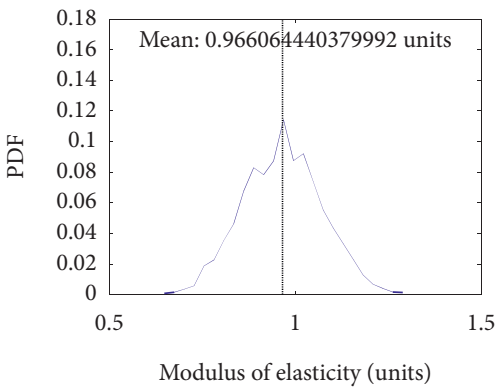

Frank
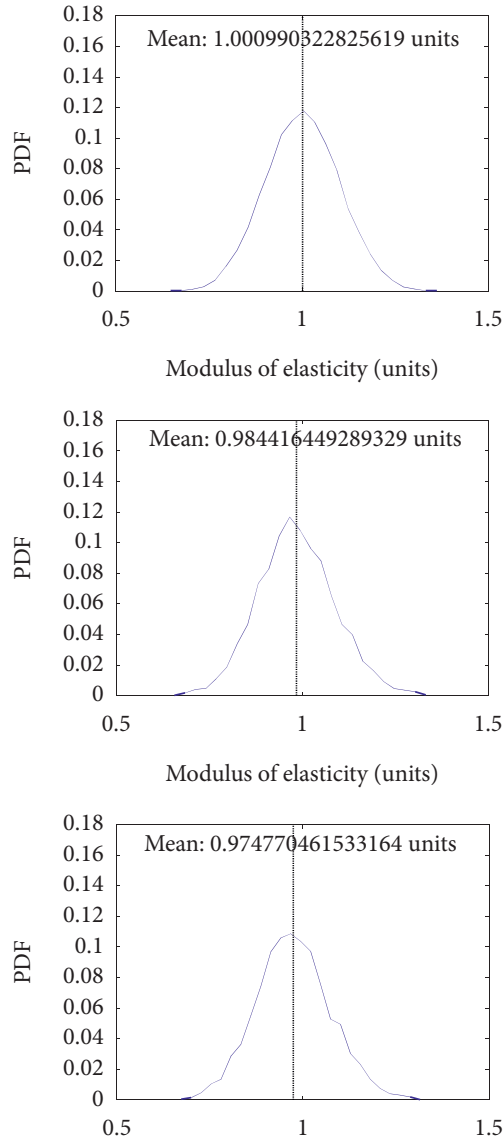

Modulus of elasticity (units)

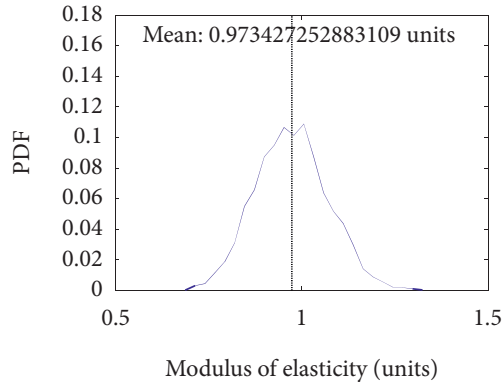

FIGURE 12: Distribution of modulus of elasticity at insignificant section: 0.9375 unit.

The great impact of dependency on distribution of conditional samples at significant section is obvious.

\section{Concluding Remarks}

The investigation to the uncertainties of low carbon steel properties demonstrates the possible nonlinear dependencies in practice. However, the incorporation of this nonlinearity into reliability analysis under the ubiquitous linear correlations is not addressed. This paper extends the subset simulation to structural reliability involving nonlinear dependencies and investigates its effect. The vine copula model is adopted to allow for modeling of multiple nonlinear dependencies, while the model parameters are estimated from linear correlations by a sequential search strategy. The algorithm for model parameter searching is optimized so that high-dimensional reliability problems involving time or spatial variance can be handled efficiently.

The deviation in failure probability due to different dependencies is generally trivial if the expected failure probability tends to be large. However, structural systems are usually designed towards high reliability, where the deviation can increase significantly. For spatially variable parameters, the deviation is more sensitive to the correlation length except for highly ragged or smooth random fields. The deviation in failure probabilities corresponding to different dependencies can reach to a thousand of times if the spatial variation is neither drastic nor smooth. 
Unfortunately, the correlation length in practice is commonly somewhere between the two extreme cases, which highlighted the importance of dependence modeling.

The accurate modeling of multivariate distributions requires statistical fitting from substantial experimental data, which is not always fulfilled in practice. Instead, the statistical moments such as means, variance, and covariance can be easily obtained from available information. Therefore, a feasible way is to determine the dependency qualitatively from the limited data. Then, the reliability can be quantitatively assessed by integrating the best-fit copulas with given statistics of each uncertain parameter.

\section{Notation}

$\begin{array}{ll}A: & \text { Cross-sectional area } \\ b: & \text { Correlation length } \\ C: & \text { Copula function } \\ c: & \text { Copula density function } \\ D: & \text { Diameter } \\ E: & \text { Modulus of elasticity } \\ E x: & \text { Expectation operator } \\ f(x): & \text { Marginal PDF } \\ F(x): & \text { Marginal CDF } \\ f_{\mathbf{X}}(\mathbf{X}): & \text { Joint PDF } \\ F_{i}: & \text { Failure event } \\ G(\cdot): & \text { Performance function } \\ I: & \text { Moment of inertia } \\ p: & \text { Failure probability } \\ P: & \text { Load } \\ \mathbf{u}: & \text { Random variables uniformly distributed on }[0,1] \\ \mathbf{U}: & \text { Random variables in the } U \text {-space } \\ \mathbf{X}: & \text { Random vector in the original space } \\ \varphi: & \text { Standard Gaussian PDF } \\ \Phi: & \text { Standard Gaussian CDF } \\ \theta: & \text { Parameter of bivariate copula function } \\ \rho: & \text { Linear correlation coefficient } \\ \mu: & \text { Mean } \\ \sigma: & \text { Standard deviation } \\ \Gamma: & \text { Autocovariance function } \\ \varepsilon: & \text { Displacement threshold value. }\end{array}$

\section{Data Availability}

The data used to support the findings of this study are included within the article.

\section{Conflicts of Interest}

The authors declare that they have no conflicts of interest.

\section{Acknowledgments}

This research was supported by the National Natural Science Foundation of China (NSFC), under Grant no. 51608399, and "Jiangsu Smart Factory Engineering Research Center (Huaiyin Institute of Technology)," under Grant no. JSFE1903. The authors gratefully acknowledge the assistance of Dr. Fan Wang for his support in the calculation.

\section{References}

[1] J. Christian and G. Baecher, Reliability and Statistics in Geotechnical EngineeringJohn Wiley \& Sons, Chichester, UK, 2003.

[2] J. Bauer and W. Puła, "Reliability with respect to settlement limit-states of shallow foundations on linearly-deformable subsoil," Computers and Geotechnics, vol. 26, no. 3, pp. 281-308, 2000.

[3] Y. Zhang, M. Beer, and S. T. Quek, "Long-term performance assessment and design of offshore structures," Computers \& Structures, vol. 154, pp. 101-115, 2015.

[4] W. Han, G. Zhou, D. Gao et al., "Experimental analysis of the pore structure and fractal characteristics of different metamorphic coal based on mercury intrusion-nitrogen adsorption porosimetry," Powder Technology, vol. 362, pp. 386-398, 2020.

[5] W. Han, G. Zhou, M. Xing et al., "Experimental investigation on physicochemical characteristics of coal treated with synthetic sodium salicylate-imidazole ionic liquids," Journal of Molecular Liquids, vol. 327, Article ID 114822, 2021.

[6] H. Pradlwarter and G. Schuëller, "Uncertain linear structural systems in dynamics: efficient stochastic reliability assessment," Computers \& Structures, vol. 88, no. 1, pp. 74-86, 2010.

[7] C. Wang, H. Zhang, and Q. Li, "Reliability assessment of aging structures subjected to gradual and shock deteriorations," Reliability Engineering \& System Safety, vol. 161, pp. 78-86, 2017.

[8] Z. Wang and W. Chen, "Time-variant reliability assessment through equivalent stochastic process transformation," Reliability Engineering \& System Safety, vol. 152, pp. 166-175, 2016.

[9] Z. Wang and W. Chen, "Confidence-based adaptive extreme response surface for time-variant reliability analysis under random excitation," Structural Safety, vol. 64, pp. 76-86, 2017.

[10] Q. Ge and M. Menendez, "Extending Morris method for qualitative global sensitivity analysis of models with dependent inputs," Reliability Engineering \& System Safety, vol. 162, pp. 28-39, 2017.

[11] D.-S. Kim, S.-Y. Ok, J. Song, and H.-M. Koh, "System reliability analysis using dominant failure modes identified by selective searching technique," Reliability Engineering \& System Safety, vol. 119, pp. 316-331, 2013.

[12] R. Lebrun and A. Dutfoy, "Do Rosenblatt and Nataf isoprobabilistic transformations really differ?" Probabilistic Engineering Mechanics, vol. 24, no. 4, pp. 577-584, 2009.

[13] R. B. Nelsen, An Introduction to Copulas, Springer, New York, NY, USA, 2nd edition, 2006.

[14] D. A. Alvarez, J. E. Hurtado, and J. Ramírez, "Tighter bounds on the probability of failure than those provided by random set theory," Computers \& Structures, vol. 189, pp. 101-113, 2017.

[15] Á. Rózsás and Z. Mogyorósi, "The effect of copulas on timevariant reliability involving time-continuous stochastic processes," Structural Safety, vol. 66, pp. 94-105, 2017.

[16] X.-S. Tang, D.-Q. Li, G. Rong, K.-K. Phoon, and C.-B. Zhou, "Impact of copula selection on geotechnical reliability under incomplete probability information," Computers and Geotechnics, vol. 49, pp. 264-278, 2013.

[17] X.-S. Tang, D.-Q. Li, C.-B. Zhou, and K.-K. Phoon, "Copulabased approaches for evaluating slope reliability under incomplete probability information," Structural Safety, vol. 52, pp. 90-99, 2015. 
[18] A. Dutfoy and R. Lebrun, "Practical approach to dependence modelling using copulas," Proceedings of the Institution of Mechanical Engineers-Part O: Journal of Risk and Reliability, vol. 223, no. 4, pp. 347-361, 2009.

[19] S. Ferson and J. G. Hajagos, "Varying correlation coefficients can underestimate uncertainty in probabilistic models," Reliability Engineering \& System Safety, vol. 91, no. 10, pp. 1461-1467, 2006.

[20] F. Wang and H. Li, "Towards reliability evaluation involving correlated multivariates under incomplete probability information: a reconstructed joint probability distribution for isoprobabilistic transformation," Structural Safety, vol. 69, pp. 1-10, 2017.

[21] F. Wang and H. Li, "The role of copulas in random fields: characterization and application," Structural Safety, vol. 75, pp. 75-88, 2018a.

[22] F. Wang and H. Li, "System reliability under prescribed marginals and correlations: are we correct about the effect of correlations?" Reliability Engineering \& System Safety, vol. 173, pp. 94-104, 2018.

[23] International Organization for Standardization, Structural Steels-Part 1: General Technical Delivery Conditions for HotRolled Products, International Organization for Standardization, Geneva, Switzerland, ISO 630-1:2011), 1st edition, 2011.

[24] K. Aas, C. Czado, A. Frigessi, and H. Bakken, "Pair-copula constructions of multiple dependence," Insurance: Mathematics and Economics, vol. 44, no. 2, pp. 182-198, 2009.

[25] H. Joe, "Families of $\$ \mathrm{~m} \$$-variate distributions with given margins and $\$ \mathrm{~m}(\mathrm{~m}-1) / 2 \$$ bivariate dependence parameters," Lecture Notes-Monograph Series, vol. 28, pp. 120-141, 1996.

[26] T. Bedford and R. M. Cooke, "Vines: a new graphical model for dependent random variables," Annals of Statistics, vol. 30, pp. 1031-1068, 2002.

[27] B. Biller and C. Gunes Corlu, "Copula-based multivariate input modeling," Surveys in Operations Research and Management Science, vol. 17, no. 2, pp. 69-84, 2012.

[28] A. H.-S. Ang and W. H. Tang, Probability Concepts in Engineering Planning and Design: Emphasis on Application to Civil and Environmental Engineering, Wiley, Hoboken, NJ, USA, 2007.

[29] O. Ditlevsen and H. O. Madsen, Structural Reliability Methods, Vol. 178, Wiley, New York, NY, USA, 1996.

[30] G. I. Schuëller, H. J. Pradlwarter, and P. S. Koutsourelakis, “A critical appraisal of reliability estimation procedures for high dimensions," Probabilistic Engineering Mechanics, vol. 19, no. 4, pp. 463-474, 2004.

[31] G. I. Schuëller and H. J. Pradlwarter, "Benchmark study on reliability estimation in higher dimensions of structural systems-an overview," Structural Safety, vol. 29, no. 3, pp. 167-182, 2007.

[32] S.-K. Au and Y. Wang, Engineering Risk Assessment with Subset Simulation, John Wiley \& Sons, Hoboken, NJ, USA, 2014.

[33] K. F. Tee, L. R. Khan, and H. Li, "Application of subset simulation in reliability estimation of underground pipelines," Reliability Engineering \& System Safety, vol. 130, pp. 125-131, 2014.

[34] K. M. Zuev, J. L. Beck, S.-K. Au, and L. S. Katafygiotis, "Bayesian post-processor and other enhancements of Subset Simulation for estimating failure probabilities in high dimensions," Computers \& Structures, vol. 92-93, pp. 283-296, 2012.
[35] M. Rosenblatt, "Remarks on a multivariate transformation," The Annals of Mathematical Statistics, vol. 23, no. 3, pp. 470-472, 1952.

[36] C. G. Bucher and U. Bourgund, "A fast and efficient response surface approach for structural reliability problems," Structural Safety, vol. 7, no. 1, pp. 57-66, 1990.

[37] X. S. Nguyen, A. Sellier, F. Duprat, and G. Pons, "Adaptive response surface method based on a double weighted regression technique," Probabilistic Engineering Mechanics, vol. 24, no. 2, pp. 135-143, 2009.

[38] W. Zhao, F. Fan, and W. Wang, "Non-linear partial least squares response surface method for structural reliability analysis," Reliability Engineering \& System Safety, vol. 161, pp. 69-77, 2017.

[39] H. Joe, H. Li, and A. K. Nikoloulopoulos, "Tail dependence functions and vine copulas," Journal of Multivariate Analysis, vol. 101, no. 1, pp. 252-270, 2010.

[40] H. Dai, G. Xue, and W. Wang, "An adaptive wavelet frame neural network method for efficient reliability analysis," Computer-Aided Civil and Infrastructure Engineering, vol. 29, no. 10, pp. 801-814, 2014.

[41] H. Dai, H. Zhang, and W. Wang, "A multiwavelet neural network-based response surface method for structural reliability analysis," Computer-Aided Civil and Infrastructure Engineering, vol. 30, no. 2, pp. 151-162, 2015.

[42] R. G. Ghanem and P. D. Spanos, Stochastic Finite Elements: A Spectral Approach, Courier Corporation, Chelmsford, MA, USA, 2003.

[43] D. Xiu, Numerical Methods for Stochastic Computations: A Spectral Method Approach, Princeton University Press, Princeton, NJ, USA, 2010.

[44] P. Sasikumar, R. Suresh, P. K. Vijayaghosh, and S. Gupta, "Experimental characterisation of random field models for CFRP composite panels," Composite Structures, vol. 120, pp. 451-471, 2015. 